\title{
Pressure-Tuned Interactions in Frustrated Magnets: Pathway to Quantum Spin Liquids?
}

\author{
Tobias Biesner *(D) and Ece Uykur \\ 1. Physikalisches Institut, Universität Stuttgart, Pfaffenwaldring 57, 70550 Stuttgart, Germany; \\ ece.uykur@pi1.physik.uni-stuttgart.de \\ * Correspondence: tobias.biesner@pi1.physik.uni-stuttgart.de
}

Received: 30 October 2019; Accepted: 21 November 2019; Published: 18 December 2019

\begin{abstract}
Quantum spin liquids are prime examples of strongly entangled phases of matter with unconventional exotic excitations. Here, strong quantum fluctuations prohibit the freezing of the spin system. On the other hand, frustrated magnets, the proper platforms to search for the quantum spin liquid candidates, still show a magnetic ground state in most of the cases. Pressure is an effective tuning parameter of structural properties and electronic correlations. Nevertheless, the ability to influence the magnetic phases should not be forgotten. We review experimental progress in the field of pressure-tuned magnetic interactions in candidate systems. Elaborating on the possibility of tuned quantum phase transitions, we further show that chemical or external pressure is a suitable parameter in these exotic states of matter.
\end{abstract}

Keywords: quantum spin liquids; frustrated magnets; quantum phase transitions; high-pressure measurements

\section{Introduction}

Quantum spin liquids (QSLs) possess nontrivial ground states, where a local order parameter does not exist. Moreover, it is not possible to observe spontaneous symmetry breaking even at very low temperatures. It is often thought that the QSLs are associated with topological phase transitions $[1,2]$. This make these systems a point of interest, and experimental evidences of this state are one of the central topics in the condensed matter physics.

QSLs are discussed in the framework of strongly correlated electron systems, while they are Mott insulators with half-filled electronic bands, and the electron-electron correlations play an important role. Possessing rich physics and properties, QSLs are subject to extensive experimental and theoretical efforts. This also bring the search for candidate materials, especially in 2D or 3D. The geometrically frustrated materials, where the resonating valance bond (RVB) model [3] is applicable, and the Kitaev QSL candidates, where the Kitaev physics [4] is relevant, are two groups of materials of which the candidates are searched for.

Technically speaking, it is difficult to identify the QSL state, as one needs to reach absolute zero temperature that is not achievable. Therefore, within the experimentally reachable limits, temperatures far below (2-3 orders of magnitude) the temperature that identify the magnetic exchange coupling (preferably antiferromagnetic (AFM) spin interactions) are assumed to show properties at the zero-temperature limits. The first step is to deduce the magnetic exchange coupling constant from the high-temperature behavior of the material via magnetic susceptibility measurements. To identify a QSL state, it is crucial to verify that there is no magnetic ordering or spin freezing down to very low temperatures. Magnetic susceptibility measurements are used initially to check the condition, where the absence (or existence) of a magnetic ordering can be identified. Absence of a sharp $\lambda$-type peak in specific heat vs. temperature curves is another indication of the absence of a magnetic ordering, 
albeit exceptions exist in the case of topological phase transitions [5]. Specific heat is a useful probe; while it can give insight to the absence of a long-range magnetic order, examination of the entropy release at the measured temperature can also help to estimate the possibility of the system to establish a long-range magnetic order at low temperatures. Beside the above mentioned macroscopic probes, more local probes such as muon spin relaxation and nuclear magnetic resonance are usually in play to detect the possible spin freezing or order. Neutron diffraction is also used to detect magnetic ordering.

While the absence of the long-range magnetic ordering is the first step to check, it is still not very satisfactory to establish a system as a QSL, while in principle, disorder effects can also give rise to such ground states without a long-range magnetic ordering. Another aspect that defines the QSL state is the fractional spin excitations, which might also be the key point to identifying this state more confidently. For instance, spinons predicted within the RVB model are worth seeking. They are fermionic quasiparticles carrying fractional spins with their own dispersion expected to give low-lying excitations and can be eventually used to identify the QSL state.

This review will mainly focus on the inorganic systems of the frustrated lattices, such as pyrochlore, triangular, honeycomb, and kagome compounds. Several review articles are already written on this topic [6-11], while this particular one aims to bring together the published works and to present the ongoing discussion of the QSL state emerging under high pressure. However, owing to the fact that the QSLs are Mott insulators and in light of theoretical proposals [12] that they are the parent states of the high-temperature superconductivity, organic conductors also are promising candidates to search for. Moreover, external pressure has already been successfully used to tune these systems to a superconducting state, albeit the high-temperature superconductivity could not be achieved. Although, it is still experimentally challenging to prove whether the Mott insulator ground state is QSL, the lack of magnetic ground states have been reported by nuclear magnetic resonance (NMR) measurements for several organic charge transfer salts. The QSL state and its evolution with external and/or chemical pressure have been discussed extensively in several review articles [13,14].

In this review, we would like to discuss the search of QSL state from another perspective, from a rather indirect route. Within the search of QSL candidates, many others also come into light that eventually are proven to be not a QSL. On the other hand, they already are very close to the conditions in which are searched for the realization of the QSL state. Perhaps a fine tuning in certain parameters, such as magnetic exchange interactions, lattice parameters, etc., can push these closer to the QSL state. Here, we look into the external pressure as this tuning mechanism. Pressure is generally accepted to be a clean tuning parameter of structural properties and electronic correlations. While it can be compared to the chemical doping effects in some cases, it allows one to eliminate the additional disorder introduced via chemical doping. In this review, we want to discuss the recent progress of the high-pressure studies, especially on the frustrated magnets, systems that are often studied in the search of the QSL candidates. We want to focus on the following questions: How does the pressure affect the frustrated systems? Which phases can we tune? Can we tune magnetic interactions directly? How do chemical and external pressure differ? Finally, can we use external pressure as a pathway to realize QSLs?

\section{Pyrochlore Lattice}

The pyrochlore lattice is a prime example for frustrated magnetism in three dimensions. While classical spin ice states are realized in $\mathrm{Ho}_{2} \mathrm{Ti}_{2} \mathrm{O}_{7}$ and $\mathrm{Dy}_{2} \mathrm{Ti}_{2} \mathrm{O}_{7}[15,16], \mathrm{Yb}_{2} \mathrm{Ti}_{2} \mathrm{O}_{7}$ is a candidate system for a quantum spin ice ground state. Here, the magnetic monopoles, obeying an ice rule, become long-range entangled. Necessary conditions for such a quantum mechanical state are small spin quantum numbers and quantum fluctuations within the degenerated ground state manifold. $\mathrm{Yb}_{2} \mathrm{Ti}_{2} \mathrm{O}_{7}$ hosts a minimal $S=1 / 2$ spin of the crystal field Kramers doublet $\left(\mathrm{Yb}_{3}^{+11}\right)$. Strong quantum fluctuations are mediated by anisotropic exchange interactions and an XY g-tensor [17]. Considering the magnetic ground state, a sample-dependency possibly induced by disorder (e.g., excess magnetic ions in the stuffed pyrochlore lattice) might explain different reported results. In particular, ordered ferromagnetic 
ground states were reported for single-crystal samples, while polycrystalline powders showed no indication of a spin freezing. Kermarrec et al. [17] combined muon spin relaxation ( $\mu$ SR) and neutron diffraction measurements under pressure to explore the low-temperature ground state of $\mathrm{Yb}_{2+x} \mathrm{Ti}_{2-x} \mathrm{O}_{7}$. Figure 1 a shows a pressure-dependent phase diagram. Upon cooling, the paramagnetic state vanishes and most of the $\mathrm{Yb}$ magnetic moments were found to be in a fast fluctuating regime even down to low temperatures, reminiscent of a QSL state. Under hydrostatic pressure, pristine samples undergo a transition from this nonmagnetic ground state to a splayed ice-like ferromagnet (the magnetic moments are sketched in Figure 1a). Figure 1 b shows the pressure dependency of a developing magnetic fraction upon cooling, leading to the magnetically ordered phase. By applying pressure a freezing of magnetic moments, increasing the magnetic fractions is observed. Additionally, the freezing temperature (defined as a fraction of $50 \%$ frozen out magnetic moments) increases with pressure. In contrast, in the stuffed compound ( $x=0.046)$, no transition is observed up to the maximal pressure of $2.41 \mathrm{GPa}$. This study shows how fragile the balance of anisotropic exchange in the quantum spin ice Hamiltonian on the pyrochlore lattice can be against external or chemical pressure. Remarkably, the lattice structure is not expected to change noticeably in the low-pressure range. A structural phase transition is observed only above $29 \mathrm{GPa}$ [18]. This illustrates that pressure is an effective tool to tune magnetic exchange interactions directly.

Chemical pressure on $\mathrm{Yb}_{2} \mathrm{X}_{2} \mathrm{O}_{7}(X=\mathrm{Sn}, \mathrm{Ti}, \mathrm{Ge})$ was probed on polycrystalline samples [19]. While the $X=\mathrm{Ti}$ and $\mathrm{Sn}$ samples order into a ferromagnet at $0.13 \mathrm{~K}$ and $0.25 \mathrm{~K}$, respectively, $\mathrm{Yb}_{2} \mathrm{Ge}_{2} \mathrm{O}_{7}$ exhibits an antiferromagnetic ground state below $0.62 \mathrm{~K}$. Different to the physical pressure, the lattice parameter increases for the Sn compound and decreases for the smaller $\mathrm{Ge}^{4+}$ ion. In general, a decreasing Curie-Weiss temperature was found with increasing lattice parameter.

Note that there are other high-pressure studies on the pyrochlore lattice $\left(\mathrm{A}_{2} \mathrm{~B}_{2} \mathrm{O}_{7}, \mathrm{~A}=\mathrm{Eu}, \mathrm{Dy}\right.$; $\mathrm{B}=\mathrm{Ti}, \mathrm{Zr})[20]$.
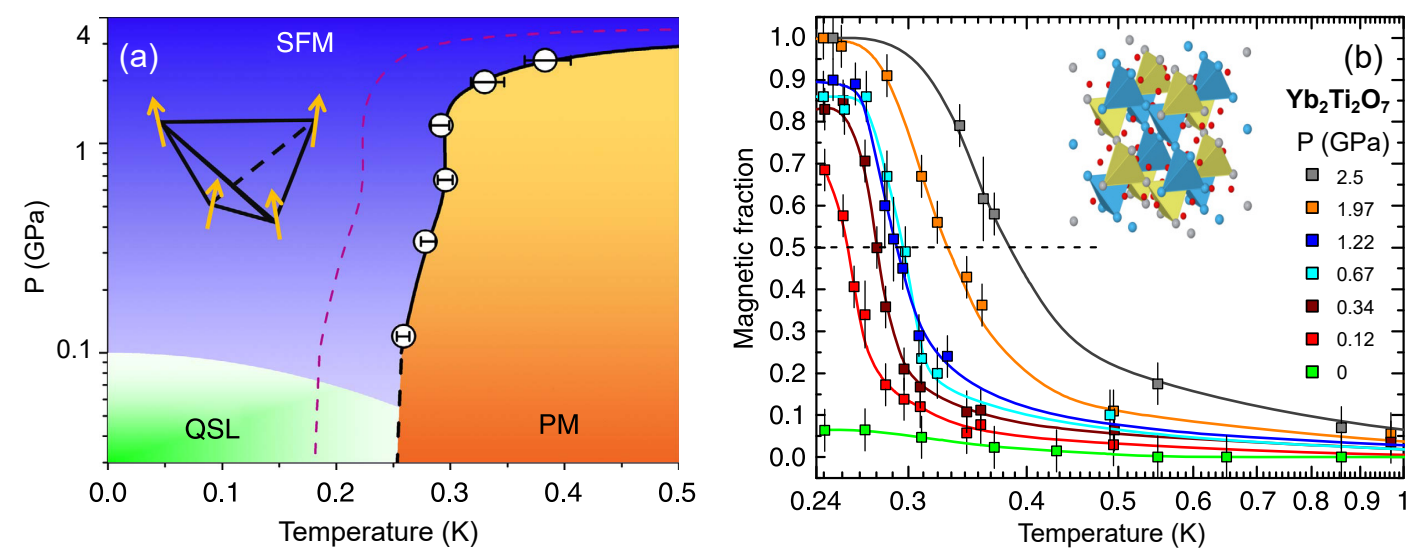

Figure 1. Results of $\mu \mathrm{SR}$ experiments on $\mathrm{Yb}_{2+x} \mathrm{Ti}_{2-x} \mathrm{O}_{7}$ : (a) The pressure-dependent phase diagram hosts three phases in the experimental accessed range. Under ambient pressure, the compound shows a ground state with large quantum fluctuations reminiscent of a quantum spin liquid (QSL) which freezes under pressure in favour of a canted ferromagnet. The dashed purple line marks the hypothetical transition for the stuffed compound $x=0.046$. (b) As the ordered phase is approached, the magnetic fraction gets enhanced due to pressure. The freezing temperature (dashed line) is furthermore increased at the high-pressure side. Graphs are reproduced from Reference [17]. 
2.1. $\mathrm{Tb}_{2} \mathrm{Ti}_{2} \mathrm{O}_{7}$
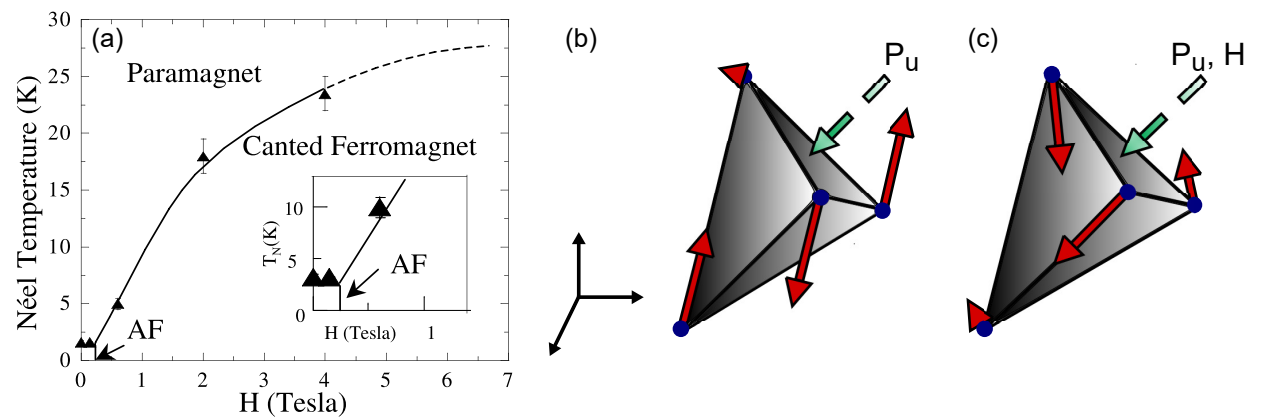

Figure 2. (a) The magnetic phase diagram of single crystalline $\mathrm{Tb}_{2} \mathrm{Ti}_{2} \mathrm{O}_{7}$ under hydrostatic pressure $P_{i}=2.4 \mathrm{GPa}$ and uniaxial stress $P_{u}=0.3 \mathrm{GPa}$ : Under pressure, an ordered AFM ground state forms below $T_{N}$. With a magnetic field of $0.6 \mathrm{~T}$, the AFM order gets lifted in favour of a canted ferromagnet. (b) Spin structure of $\mathrm{Tb}$ tetrahedron under pressure: The AFM structure (red arrows) is formed under a pressure of $P_{i}=2.4 \mathrm{GPa}$ and uniaxial stress $P_{u}=0.3 \mathrm{GPa}$ along the [011] axis (green arrow). (c) Canted ferromagnet for an additional field $H=4 \mathrm{~T}$ parallel to $P_{u}$ : Blue spheres indicate Tb sites; principal axes of the cubic cell consisting of $4 \mathrm{~Tb}$ tetrahedra are indicated by black arrows. Graphs are reproduced from Reference [21].

The low-temperature ground state of the spin liquid compound $\mathrm{Tb}_{2} \mathrm{Ti}_{2} \mathrm{O}_{7}$ has been extensively studied but remains intriguing. With an antiferromagnetic Curie-Weiss temperature of $\theta_{C W}=-13 \mathrm{~K}$ (respectively $-19 \mathrm{~K}$, including crystal field contribution) and Ising-like $5 \mu_{b} \mathrm{~Tb}^{3+}$ spins of the crystal-field doublet, the compound, however, shows no onset of static order down to at least $43 \mathrm{mK}$ [22-26]. Interestingly, antiferromagnetic short-range correlations are set below $50 \mathrm{~K}$ [22]. Various suggestions to explain the missing Néel state have been given (see Reference [27] and references herein): a quantum spin ice state [28], structural distortion [29], or magnetoelastic excitations [30]. Mirebeau et al. extensively investigated the enigmatic nonmagnetic ground state by disturbing it by means of pressure and magnetic field [21,31-33]. Here, pressure is used to destabilize the balance of superexchange, crystal-field interactions, and dipolar coupling between neighbouring $\mathrm{Tb}^{3+}$ cations. With a decreasing lattice constant of $1 \%$ at $8.6 \mathrm{GPa}$ and preserved $F d \overline{3} m$ symmetry, the effect of pressure on the crystal structure was found to be rather small [31,34]. Neutron diffraction on polycrystalline samples under pressure reveals a complex antiferromagnetic structure below $T_{N}=2.1 \mathrm{~K}$, coexisting with the spin liquid ground state. Bragg peaks of this antiferromagnetic structure are observed for a surprisingly low pressure of $1.5 \mathrm{GPa}$ [31]. Interestingly, the Néel temperature seems to be insensitive to hydrostatic pressure but depends on applied strain [21,32]. Moreover, experiments on single crystals [21] show that a combination of isotropic and uniaxial pressures is crucial for disturbing the spin liquid ground state since hydrostatic pressure alone does not introduce a magnetic order in the single crystals.

Combining a magnetic field parallel to uniaxial stress of $P_{u}=0.3 \mathrm{GPa}$ along the [011] direction and an isotropic pressure component of $P_{i}=2.4 \mathrm{GPa}$ leads to suppression of the antiferromagnetic structure in favor of a canted ferromagnet (see the phase diagram in Figure 2a). Simultaneously, $T_{N}$ gets increased under magnetic field. Mirebeau et al. argue that, while the dipolar interaction is only weakly affected by pressure, superexchange is strongly influenced. The effect of pressure on the spin structure of the $\mathrm{Tb}$ tetrahedron is shown in Figure $2 \mathrm{~b}, \mathrm{c}$, with and without magnetic field parallel to $P_{u}$, respectively. Here, the isotropic compound increases exchange energy about $\frac{d J}{J d P}=0.07 \mathrm{GPa}^{-1}$. A uniaxial strain along the [011] axis lifts the geometrical frustration by compressing $1 / 3 \mathrm{of} \mathrm{Tb}-\mathrm{Tb}$ bonds of about $0.3 \%$ (for $P=0.3 \mathrm{GPa}$ ) and by decreasing the remaining $2 / 3$ by about $0.1 \%$. Due to this lifting of frustration, magnetic order is introduced. With increase of magnetic field, the spins are further reoriented in the canted ferromagnetic structure (Figure 2c). 
Compared to physical pressure, the effect of chemical pressure on the ground state of $\mathrm{Tb}_{2} \mathrm{Ti}_{2} \mathrm{O}_{7}$ is different [27,33,35]. A negative chemical pressure for the case of replacing titanium by the larger tin leads to lattice expansion. Despite antiferromagnetic interactions at higher temperatures leading to a Curie-Weiss temperature between -11 to $-12 \mathrm{~K}$, the compound shows a ferromagnetic contribution in $37 \%$ of the $\mathrm{Tb}^{3+}$ spins below a transition temperature of $0.87 \mathrm{~K}$. Together with an antiferromagnetic "two in, two out" ice rule, these ferromagnetic domains form the magnetic ground state. Possibly, the compound orders because of the weakened antiferromagnetic exchange compared to the pure $\mathrm{Tb}_{2} \mathrm{Ti}_{2} \mathrm{O}_{7}$ sample and because of a distortion of the local crystal field [33]. A positive chemical pressure is reached by substituting titanium with germanium, leading to a smaller lattice constant [27]. The contraction was found to be larger ( $2 \%$ ) compared to a physical pressure of $8.6 \mathrm{GPa}$ (1\% cf. Reference [21]). The reduced $\mathrm{Tb}-\mathrm{Tb}$ distance results in a stronger antiferromagnetic exchange as indicated by a higher Curie-Weiss temperature of $-19.2 \mathrm{~K}$ compared to Ti and Sn compounds. Different from the physical counterpart, positive chemical pressure here induces short-ranged ferromagnetic correlations, coexisting with the liquid-like correlations, as observed by neutron scattering [27]. Similar to $\mathrm{Tb}_{2} \mathrm{Ti}_{2} \mathrm{O}_{7}$, no long-range order is observed down to $20 \mathrm{mK}$.

\subsection{Dichalcogenides}

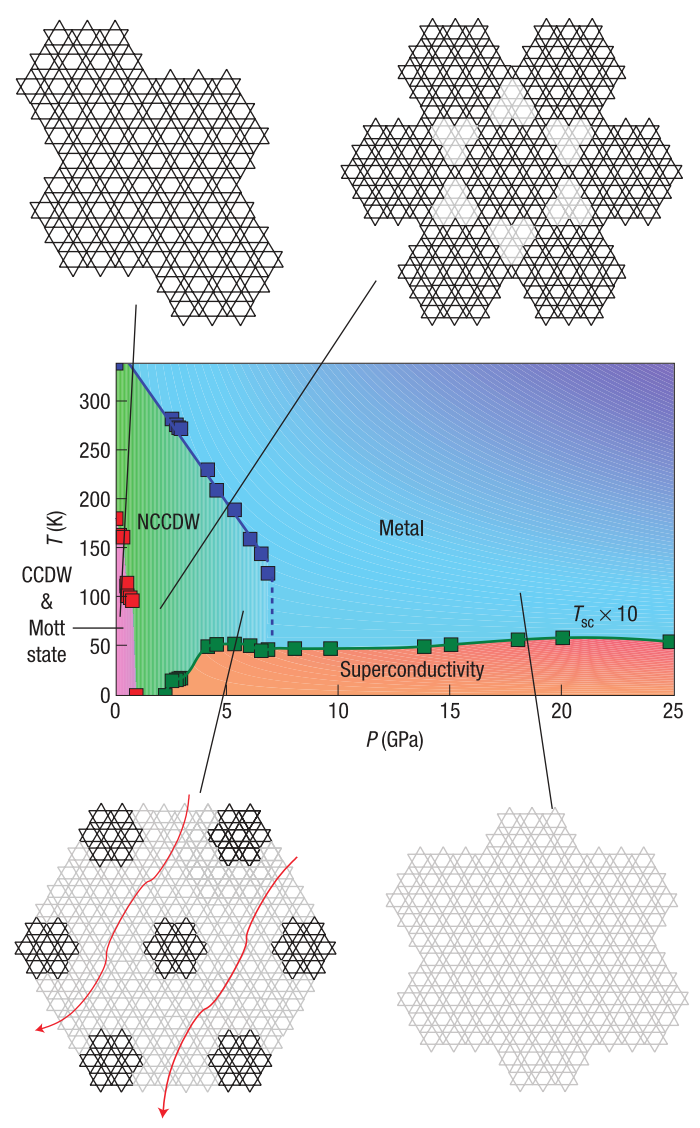

Figure 3. Phase diagram of $1 \mathrm{~T}-\mathrm{TaS}_{2}$ under pressure: Pressure decreases the swelling of the planes related to the David-star pattern (sketched above and below the diagram). In the light grey areas, deformations are reduced or completely suppressed. Mott phase and CCDW state are suppressed over $0.8 \mathrm{GPa}$, as the NCCDW state stabilizes between 1-7 GPa. Here, hexagonal domains are formed (see sketch). As the pressure is further increased, the superconducting state develops (with a $T_{\mathcal{C}}$ of about $5 \mathrm{~K}$ ). Superconductivity remains stable up to $25 \mathrm{GPa}$, with a metallic state above $T_{\mathcal{c}}$. Graphs are reproduced from Reference [36]. 
Transition metal dichalcogenides (TMDs) attract great attention [37]. Due to the complex interplay of charge, spin, and orbital degrees of freedom, they display a rich phase diagram ranging from charge density waves (CDW), superconductivity, Mott physics, and possibly QSLs [38].

The two-dimensional 1T- $\mathrm{TaS}_{2}$ exhibits multi charge density wave ground states [39], which can be continuously manipulated via external stimuli, such as temperature, chemical $[40,41]$ or optical doping [39], disorder [42], and hydrostatic pressure [36,43]. Under ambient pressure, the compound shows metallic behavior in the high-temperature range. Below $550 \mathrm{~K}$, an incommensurate charge density wave (ICCDW) superlattice develops. As resistivity increases, the ICCDW undergoes a transition to a nearly commensurate order (NCCDW) around $350 \mathrm{~K}$. The low temperature range is governed by Mott physics in the commensurate charge density wave (CCDW) [36]. Thus, a simple model is the Mott-Hubbard Hamiltonian:

$$
H=-\sum_{<i, j>, \sigma} t_{i, j}\left(c_{i \sigma}^{\dagger} c_{j \sigma}+\text { h.c. }\right)+U \sum_{i} n_{i}\left(n_{i}-1\right) .
$$

The first term accounts for a hopping between two adjacent lattice sites $\langle i, j\rangle$ and can be interpreted as kinetic energy. $t$ is the transfer integral, which gives the hopping probability between two sites and is therefore proportional to the atomic overlap and effectively the bandwidth $W$. The creation and annihilation operators of an electron with spin $\sigma$ at site $i$ are written as $c_{i \sigma}$ and $c_{i \sigma}^{\dagger}$, respectively. The second term introduces an on-site Coulomb repulsion $U$ if site $i$ is fully occupied.

Here, pressure acts as a natural tuning parameter of the CDW states by affecting the transfer integral, $t$, and on-site Coulomb repulsion, $U$, without introducing chemical disorder. A phase diagram reproduced from Reference [36] is shown in Figure 3. With an increase of $t$ and a decrease of $U$, Mott physics melt away at a pressure of about $1 \mathrm{GPa}$, giving rise to a transition to the NCCDW state, which persists up to $7 \mathrm{GPa}$, and finally entering to a metallic state. The high-pressure ground state (over $3 \mathrm{GPa}$ ) is superconducting ( $T_{c}$ of about $5 \mathrm{~K}$ ) at least up to $25 \mathrm{GPa}$. While the Mott state CCDW clearly competes with the superconducting region [36,43], the coexistence of NCCDW and superconductivity is intriguing. At present, a macroscopic picture of superconductivity in the NCCDW phase is not fully clear. One proposed suggestion is that the superconducting phase forms within the metallic interdomain spaces of the CDW, which become connected as the CDW domains smear out under pressure [36]. On the other hand, an XRD study under pressure [43] suggests that the distance between the CDW domains decreases while domain boundaries remain sharp, meaning no interconnected metallic regions are formed. According to this picture, the whole NCCDW structure must form a single coherent superconducting phase.

By substituting sulfur by the isovalent selenium, a Mott-insulator-to-metal transition is observed [40,41]. Recent studies [41] show a melting of the Mott state CCDW due to the formation of a pseudogap, suggesting the importance of disorder and further inducing superconductivity [40]. The superconducting ground states is then suppressed in favor of a CCDW in the 1T- $\mathrm{TaSe}_{2}$ compound.

In summary, $1 \mathrm{~T}-\mathrm{TaS}_{2-x} \mathrm{Se}_{x}$ is an interesting system to explore quantum phase transitions and various exotic states of matter. Especially, the possibility of a QSL ground state remains intriguing [38]. Optical infrared or Raman measurements at low temperatures and under pressure could give further information about the CDW phases.

\section{Triangular Lattice}

The highly frustrated triangular lattice hosts a rich phase diagram of magnetic phases and QSL ground states. Focusing on the case of a Heisenberg magnetic exchange, we want to show the tunability of magnetic phases on this lattice. The general model for an anisotropic Heisenberg antiferromanget on the triangular lattice can be written as

$$
\mathcal{H}=J \sum_{<i, j>} \mathbf{S}_{\mathbf{i}} \cdot \mathbf{S}_{\mathbf{j}}+J^{\prime} \sum_{<i, j^{\prime}>} \mathbf{S}_{\mathbf{i}} \cdot \mathbf{S}_{\mathbf{j}^{\prime}},
$$


where $J$ and $J^{\prime}$ are the magnetic exchange interactions along the horizontal and diagonal bonds, respectively, and $\mathbf{S}_{\mathbf{i}}, \mathbf{S}_{\mathbf{j}}$, and $\mathbf{S}_{\mathbf{j}}$, give the spin-1/2 operators at sites $i, j$, and $j^{\prime}$, respectively [44].

\section{1. $\mathrm{Cs}_{2} \mathrm{CuCl}_{4}$}

The two-dimensional Heisenberg magnet, $\mathrm{Cs}_{2} \mathrm{CuCl}_{4}$ [45], posses $S=1 / 2$ spins with slightly different exchange values $J$ and $J^{\prime}\left(J^{\prime} / J=0.3\right)$ along the $b$-direction (horizontal bonds) and for the interchain coupling (diagonal bonds), respectively [46]. Furthermore, Dzyaloshinskii-Moriya (DM), an interplane exchange interaction, was found to be important $[47,48]$. Below $T_{N}=0.62 \mathrm{~K}$, an incommensurate spiral ground state is formed (DM spiral), with the spiral along the $b$-axis [49]. A magnetic field was shown to lift this confinement of the spins in the $b c$-plane in favour of a commensurate coplanar AFM ground state within the $a b$-plane [48]. Recently, the tunability of the spin Hamiltonian via external pressure and magnetic field was demonstrated, combining high-pressure electron spin resonance (ESR), radio frequency susceptibility measurements [44]. Here, Heisenberg exchange is continuously enhanced under pressure, leading to an increase of $J^{\prime} / \mathrm{J}$ by $12 \%$ at $1.8 \mathrm{GPa}$, as determined by the ESR (cf. Figure 4a,b) Most importantly, the interchain coupling $J^{\prime}$ gets enhanced with increasing pressure. Due to the tuned exchange interactions, new phases are emerging under magnetic field (see Figure 4c,d). The magnetic field favors more classical phases; therefore, suppressing the DM spiral at about 2.2-2.6 T, a coplanar order is stabilized in the $a b$-plane. Due to the enhanced $J^{\prime}$, a non-coplanar frustrated phase becomes stable at around 6.9 T. The emerging magnetic anomalies at 9.2 and $9.8 \mathrm{~T}$ under a pressure of 1.8 GPa are interpreted as double-cone and single-cone order, respectively (see Reference [44] and references herein). Furthermore, the fully polarized ferromagnetic high-field phase is shifted from around $9 \mathrm{~T}$ at ambient pressure to $11.5 \mathrm{~T}$ at $1.8 \mathrm{GPa}$.

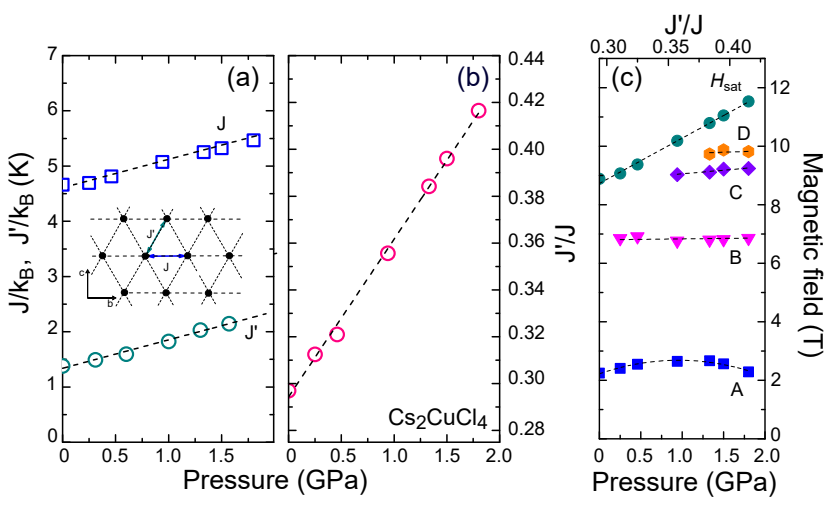

(d)

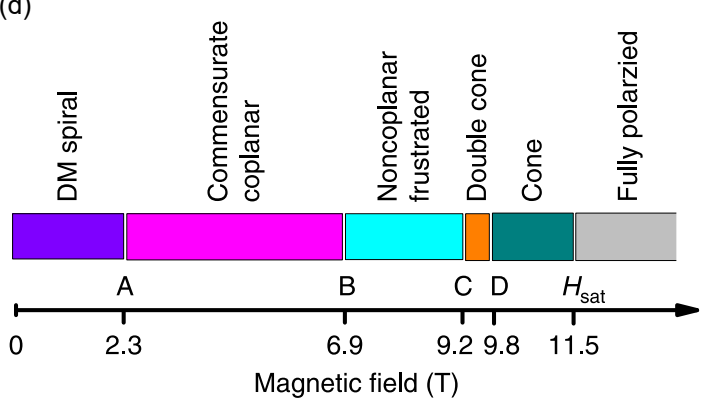

Figure 4. (a,b) Pressure-dependent magnetic exchange interactions $J$ and $J^{\prime}$ along the horizontal and diagonal bonds, respectively (see inset of the crystallographic structure): As the pressure is decreased, the Heisenberg exchange is continuously altered. (c) Simultaneously increasing a magnetic field $(H \| b)$ at $T=350 \mathrm{mK}$ unveils five different magnetic phases beside the DM spiral. (d) Proposed phase diagram under pressure (1.8 GPa) and magnetic field. Graphs are reproduced from Reference [44]. 


\section{2. $\mathrm{YbMgGaO}_{4}$}

$\mathrm{YbMgGaO}_{4}$ [50] consists of $S=1 / 2 \mathrm{Yb}^{3+}$ spins on triangular lattices, separated by nonmagnetic $\mathrm{Mg}^{2+}$ and $\mathrm{Ga}^{3+}$ ions (Figure $5 \mathrm{a}-\mathrm{c}$ ). The edge-sharing $\mathrm{YbO}_{6}$ octahedra in $R \overline{3} m$ are characterized by equal $\mathrm{Yb}-\mathrm{O}$ distances and two equal angles, the $\mathrm{Yb}-\mathrm{O}-\mathrm{Yb}$ bridging angle $\alpha$ and the $\mathrm{O}-\mathrm{Yb}-\mathrm{O}$ angle $\beta$. Structural randomness is induced by a random distribution of the $\mathrm{Mg}^{2+}$ and $\mathrm{Ga}^{3+}$ ions. The material became of recent interest as a QSL candidate, when an absence of magnetic order down to at least $50 \mathrm{mK}$ was shown [51], although possibly a weak spin freezing takes place at around $100 \mathrm{mK}$. More interesting, a magnetic continuum at low temperatures is possibly related to gapless spinons or a nearest-neighbour RVB state (see the references in Reference [52]). Focusing on combined $\mu \mathrm{SR}$, $X R D$, and DFT studies [52], we are going to review the pressure dependency of the intriguing ground state. XRD shows no changes of the crystal symmetry up to pressures as high as $10 \mathrm{GPa}$. However, the $\mathrm{Yb}-\mathrm{O}$ distances are shrinked by about $0.6 \%$ at $2.6 \mathrm{GPa}$. Importantly, the angles $\alpha$ and $\beta$ are weakly decreasing with a change of about $0.07^{\circ}$ at $2.6 \mathrm{GPa}$ and $0.2^{\circ}$ at $10 \mathrm{GPa}$. Figure $5 \mathrm{~d}$ shows the temperature dependency of the zero-field $\mu \mathrm{SR}$ spectra at ambient conditions and under pressures as high as $2.6 \mathrm{GPa}$. Of interest here is the increase of the zero-field muon relaxation rate below $4 \mathrm{~K}$. This was interpreted as the onset of spin-spin correlations, which are fully developed around $0.8 \mathrm{~K}$, leading to a dynamic spin state in $\mathrm{YbMgGaO}_{4}$. Comparing the ambient and high-pressure data, the external pressure seems to have no effect on the development of such a ground state. While comparably strong pressure-induced structural changes have been proven in other compounds to have an effect on magnetic couplings, here, the structural randomness, which is not affected by pressure, seems to be crucial in stabilizing the QSL ground state.
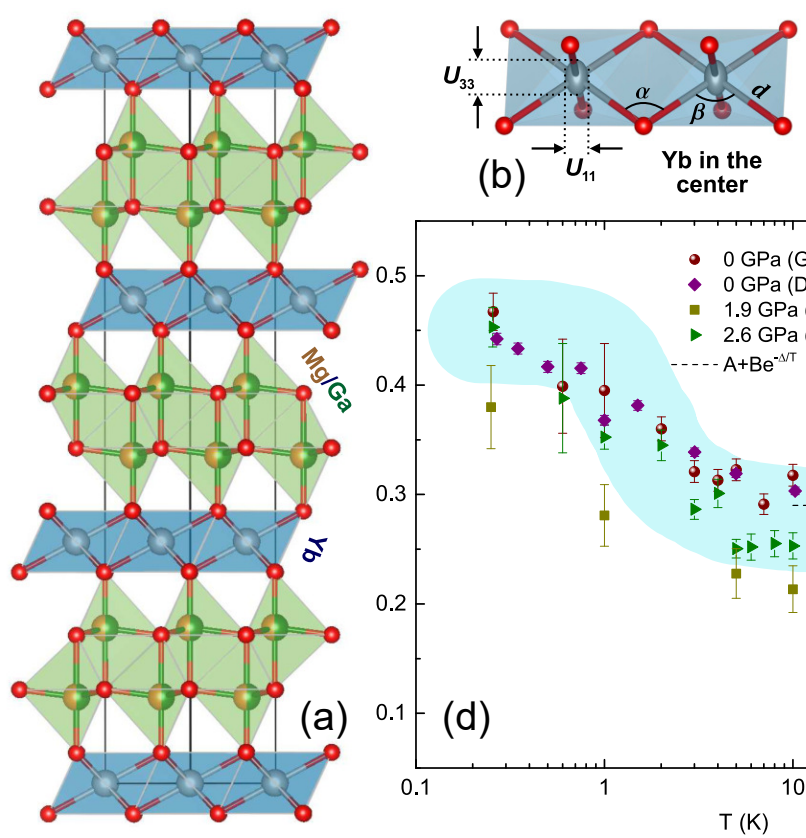

(b) $\rightarrow U_{11}^{\leftarrow} \underset{\substack{\text { Yb in the } \\ \text { center }}}{\leftarrow}$

(c)
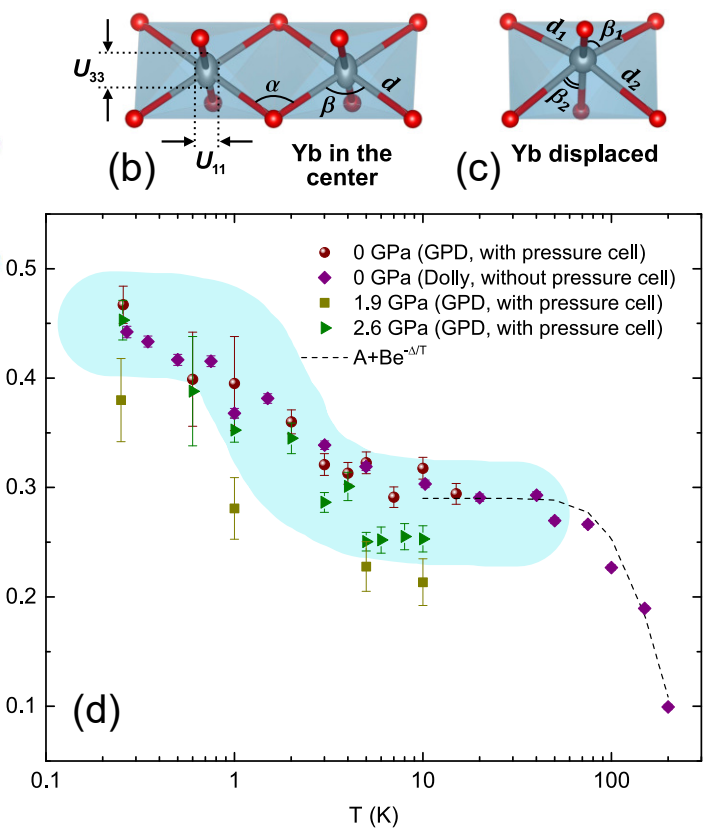

Figure 5. (a) Crystallographic structure of $\mathrm{YbMgGaO}_{4}$ : Edge-sharing $\mathrm{YbO}_{6}$ octahedra are separated by slabs of nonmagnetic $\mathrm{Mg}^{2+}$ and $\mathrm{Ga}^{3+}$ ions. (b) $\mathrm{YbO}_{6}$ octahedra with characteristic parameters. (c) Trigonal distortion of the $\mathrm{YbO}_{6}$ octahedra induced by mixing of $\mathrm{Mg}^{2+}$ and $\mathrm{Ga}^{3+}$ sides: Importantly, no changes of the structural symmetry are observed under pressure. (d) The temperature dependence of the zero-field muon relaxation rate at different pressures shows the onset of spin-spin correlations below $4 \mathrm{~K}$, fully developed at $0.8 \mathrm{~K}$. Reproduced from Reference [52].

\section{Honeycomb Lattice}

Recently frustrated magnets on the honeycomb lattice have attracted attention [8,53-55]. Among them, the Kitaev systems are of particular interest because (a) they are highly relevant for 
theoretical considerations, as Kitaev's model is directly solvable, and (b) rich physics in terms of exotic quasiparticles, e.g., Majorana fermions [56,57]. Besides the iridate systems [55], like $\mathrm{Li}_{2} \mathrm{IrO}_{3}$ and $\mathrm{Na}_{2} \mathrm{IrO}_{3}, \alpha-\mathrm{RuCl}_{3}[58,59]$ has been extensively studied.

\section{1. $\alpha-\mathrm{RuCl}_{3}$}

$\alpha-\mathrm{RuCl}_{3}$ implements the $4 \mathrm{~d}^{5}$ transition metal ruthenium $\left(\mathrm{Ru}^{3+}\right)$ with $\lambda_{\mathrm{Ru}} \approx 0.15 \mathrm{eV}[60,61]$ on a nearly ideal honeycomb lattice. It renders an edge-sharing geometry with an octahedral cage of chlorine ions. Different polymorphs with an ABC-like stacking of the $a b$ honeycomb layers along the $c$ axis $\left(\alpha-\mathrm{RuCl}_{3}\right)$, and one-dimensional face-sharing $\mathrm{RuCl}_{6}$ chains $\left(\beta-\mathrm{RuCl}_{3}\right)$ are known. Regarding the structural symmetry, different space groups $\left(P 3_{1} 12, R \overline{3}\right.$, and $\left.C 2 / \mathrm{m}\right)$ were reported (see the discussion in Reference [55]). This seems to be explained by a majority of samples showing stacking faults with a mixing of the $\mathrm{ABC}$ (rhombohedral $R \overline{3}$ ) and $\mathrm{AB}$ (monoclinic $C 2 / \mathrm{m}$ ) stacking patterns. Due to an only weak van der Waals attraction between the layers, the system is quasi-two-dimensional in the $a b$-plane and susceptible to a mixing of the two stacking patterns $[62,63]$.

Looking closer at the magnetic properties, a transition within the $a b$-plane from a paramagnetic phase, obeying a Curie-Weiss law with $\theta_{C W} \approx+40 \mathrm{~K}$ and an effective magnetic moment of about $2.3 \mu_{B}$ [64], to an antiferromagnetic zigzag order is observed [63]. As well, susceptibility measurements expose an anisotropy $\left(\chi_{a b}>\chi_{c}\right)$ [65]. While the cleanest crystals with a minimum of stacking faults show a Néel temperature of about $T_{N} \approx 7$ to $8 \mathrm{~K}, T_{N}$ is found to increase up to 10 to $14 \mathrm{~K}$ with higher amount of $\mathrm{ABC}$ and $\mathrm{AB}$ mixing, indicating different order temperatures of these patterns $[62,63]$.

Under ambient pressure, $\alpha-\mathrm{RuCl}_{3}$ is considered as a spin-orbit assisted $j_{\text {eff }}=1 / 2 \mathrm{Mott}$ insulator with a Mott gap of about $1.1 \mathrm{eV} \mathrm{[66-70].} \mathrm{Specific} \mathrm{heat} \mathrm{measurements} \mathrm{[71]} \mathrm{show} \mathrm{a} \mathrm{pressure}$ sensitivity of the Néel transition and indicate a suppression of the magnetic order at around 0.7 GPa (in accordance with the theoretical, predicted coupling constants [60]). Resistivity experiments up to 140 GPa clearly prove the persistence of the insulating state [71]. Further NMR and magnetization studies demonstrated the vanishing magnetic order under pressure together with a strongly reduced susceptibility and the absence of the low-energy fluctuations [72]. The pressure-induced state was further investigated by optical infrared spectroscopy and ab initio DFT calculations [64] and magnetization measurements combined with high-resolution x-ray diffraction experiments [73].

Here, we want to focus on the spectroscopic study of Reference [64]. First, the phononic part of the optical spectrum with the pronounced $320 \mathrm{~cm}^{-1}$ mode was investigated to elaborate on a possible structural transition under pressure. The $T=10 \mathrm{~K}$ optical conductivity is shown in Figure 6a. In addition to the main mode (peak 1, which is assigned to a trio with mostly in-plane contributions at $\omega_{0} \approx 321,322$, and $326 \mathrm{~cm}^{-1}$ ), a weaker out-of-plane mode is located at around $290 \mathrm{~cm}^{-1}$. Generally, a hardening together with a broadening of the peaks under pressure is observed. Over $0.7 \mathrm{GPa}$, the main-mode peak 1 splits while another distinct resonance (peak 2) emerges. As the pressure is increased, both peaks further experience a hardening due to lattice contraction. Since phonon modes are generally strongly dependent on the lattice symmetry, the observed splitting is a direct evidence for a symmetry breaking over a pressure of $0.7 \mathrm{GPa}$. 

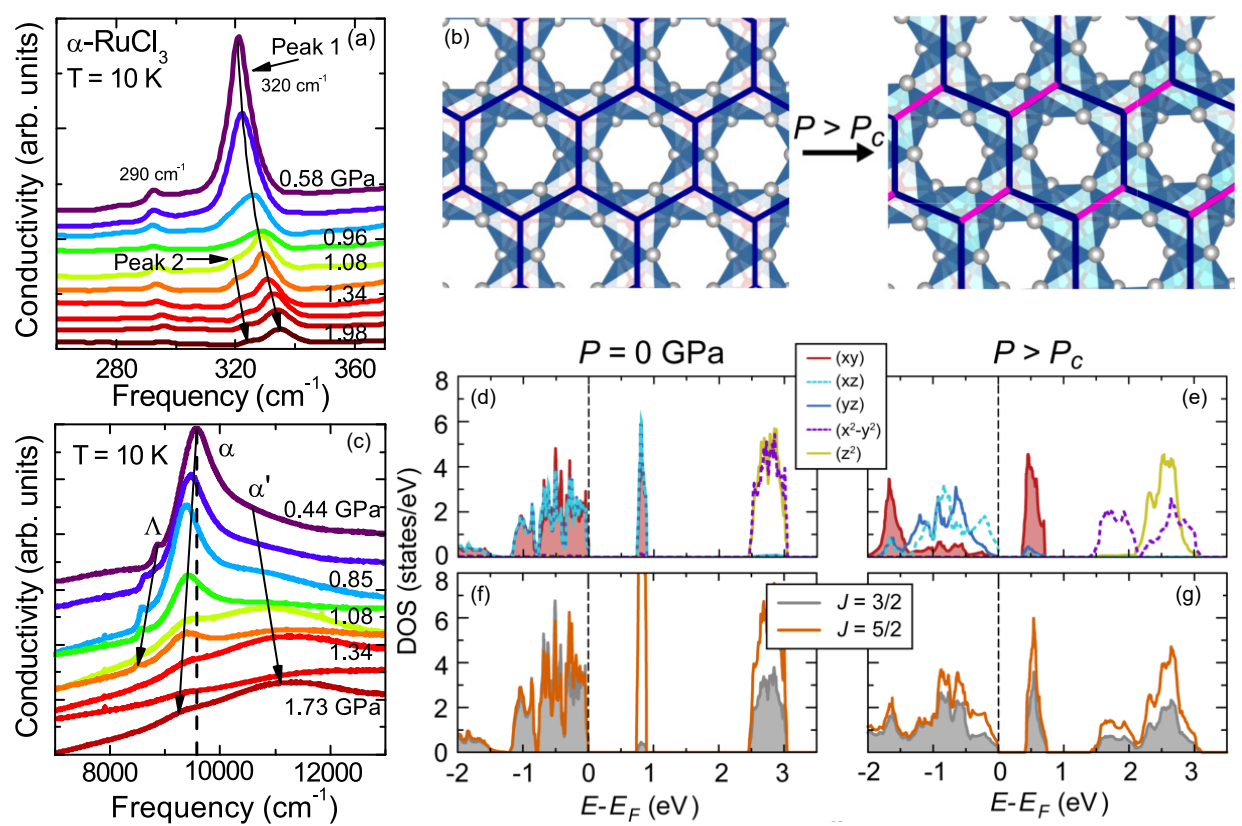

Figure 6. Pressure-dependent optical spectroscopy of $\alpha-\mathrm{RuCl}_{3}$ [64]: (a) Splitting of the phononic contributions (peak 1 ) is a direct evidence for a symmetry breaking over a pressure of $0.7 \mathrm{GPa}$. (b) The ambient pressure honeycomb (left-hand side) transforms to a dimerized structure under pressure (right-hand side). (c) The electronic ground state is studied by the pressure dependency of the Mott gap $\alpha$. As the pressure increases, the gap gets suppressed, indicating a collapse of Kitaev magnetism and a breakdown of the $j_{\text {eff }}$ picture. $(\mathbf{d}-\mathrm{g})$ Results of the GGA+SOC+U calculations, showing the breakdown of the $j_{\text {eff }}$ picture (see text for further explanations).

DFT calculations suggest that parallel dimerization of neighbouring Ru sites set in at high pressure. The homogeneous ambient-pressure honeycomb structure transforms to a triclinic $P \overline{1}$ structure (Figure 6b). As a result, a pressure-driven structural transition at $P>1 \mathrm{GPa}$ to a triclinic, dimerized structure is established by the spectroscopic experiment, well in accordance with the calculations.

To further understand the dimerized phase, the electronic part of the optical spectrum is investigated in the region of the optical gap $\alpha$ under pressure. The general suppression of the $\alpha$ peak strongly indicates a collapse of Kitaev interactions above $0.7 \mathrm{GPa}$. In this frame, a breakdown of the $j_{\text {eff }}$ picture in accordance with the suppressed magnetic susceptibility above 0.7 GPa [72] is most probable. Note that, according to transport measurements, the suppression of the $\alpha$ peak is not interpreted as a closure of the optical gap. Instead, $\alpha-\mathrm{RuCl}_{3}$ stays well in the Mott insulation region at least up to $140 \mathrm{GPa}$ [71].

The orbital-dependent density of states (DOS) was calculated including spin-orbit coupling and electronic correlations $(\mathrm{GGA}+\mathrm{SOC}+U)$ with $U=1.5 \mathrm{eV}$ giving insight to the observed optical excitations. The results are shown in Figure $6 \mathrm{~d}-\mathrm{g}$. For the undimerized structure $(P=0 \mathrm{GPa}$, left-hand side panels), the relativistic $j_{\text {eff }}$ picture is validated. In Figure $6 \mathrm{~d}$. the lower lying $t_{2 g}$ DOS with the expected orbital contributions is splitted from the higher $e_{g}$ manifold and further gapped by SOC and $U$. Thus, the narrow peak at around $1 \mathrm{eV}$ represents the single $t_{2 g}$ hole at each site residing in the upper Hubbard band. Projecting on the atomic $J$ orbitals (Figure $6 \mathrm{f}$ ) shows a mixture of the lower Hubbard band $J=5 / 2\left(j_{e f f}=1 / 2\right)$ with the $J=3 / 2\left(j_{e f f}=3 / 2\right)$ states, whereas the upper Hubbard band with mainly $J=5 / 2$ contribution is clearly distinguishable. Here, virtual hopping through the ligands via hopping channel $t_{2}$ induces a strong ferromagnetic Kitaev exchange. In the high-pressure phase ( $P>P_{c}$, right-hand side panels), the electronic density of states changes (cf. Figure $6 \mathrm{e}$ ). The $d_{x y}$ orbitals split into a bonding contribution which is lower in energy and an antibonding part at higher energy. This destroys the $j_{e f f}$, states as can be seen in the atomic projection Figure $6 \mathrm{~g}$. At the former upper Hubbard band, now a pronounced mixture of $J=3 / 2$ and $J=5 / 2$ states emerges. With this 
breakdown of the $j_{\text {eff }}$ picture, we can explain the vanishing magnetic ground state under pressures of around $1 \mathrm{GPa}[71,72]$ as a result of the formation of pseudocovalent bonds in the dimerized structure. In the high-pressure phase, the direct $\mathrm{Ru}-\mathrm{Ru}$ hopping path $t_{3}$ is enhanced along these bonds. This leads to a large antiferromagnetic Heisenberg exchange located on the dimers, further destabilizing and suppressing the magnetic low-temperature zigzag order. Consistently, the computed magnetic moments are completely suppressed in the high-pressure structure. Instead of increasing the $t_{2}$ channel towards a dominant Kitaev regime, hydrostatic pressure promotes the direct $t_{3}$ hopping. Results, therefore, conclude that the high-pressure nonmagnetic state of $\alpha-\mathrm{RuCl}_{3}$ is a valence-bond crystal and excludes a transition to a Kitaev QSL in the dimerized structure.

\subsection{Iridates}

The $5 \mathrm{~d}$ honeycomb iridates $\mathrm{A}_{2} \mathrm{IrO}_{3}(\mathrm{~A}=\mathrm{Li}, \mathrm{Na})$ [74,75] have gained attraction as Kitaev candidates $[54,55,76,77]$ and were considered in terms of the Heisenberg-Kitaev model and with additional off-diagonal contribution. Due to strong spin-orbit coupling, they are located in the relativistic Mott insulating limit, hosting $j_{\text {eff }}$ spins of the magnetic Ir ions [78,79]. Compared to $\alpha-\mathrm{RuCl}_{3}$ ( $4 \mathrm{~d}$ vs. $5 \mathrm{~d}$ electronic configuration), a stronger SOC in addition to a weaker Coulomb repulsion is generally expected. While in principle a band-insulating picture featuring quasimolecular orbitals is imaginable to explain the insulating nature of these systems [80], the Mott insulating picture is well backed by experiments and the majority of theoretical approaches. Additional trigonal crystal field splitting of the $j_{\text {eff }}=3 / 2$ quartet can be sufficient high compared to SOC. This might induce a splitting of the SO-exciton and a mixing of the $j_{\text {eff }}=3 / 2$ quartet with the $j_{\text {eff }}=1 / 2$ doublet $[78,81,82]$.

The three polymorphs of the Li-iridates are different in structure. While the $\alpha$ type is comparable to $\mathrm{Na}_{2} \mathrm{IrO}_{3}$ with a layered honeycomb lattice, the $\beta$ variant shows a more three-dimensional hyper honeycomb. The $\gamma$ type is characterized by a stripy-honeycomb structure. All types show an Ir-O-Ir edge-sharing geometry, allowing a nearly ideal $90^{\circ}$ bond. The centered $\mathrm{Ir}^{4+}$ ion is coordinated by a cage of six $\mathrm{O}^{2-}$ ions. The Ir ions generate a $5 \mathrm{~d}^{5}$ electronic structure with a single $t_{2 g}$ hole [83].

\subsection{1. $\alpha-\mathrm{Li}_{2} \mathrm{IrO}_{3}$}

The lattice structure of $\alpha-\mathrm{Li}_{2} \mathrm{IrO}_{3}$ (Figure 7a) resembles a honeycomb of edge-sharing $\mathrm{IrO}_{6}$ octahedra (Ir-O-Ir bond angles of around $95^{\circ}$ with 5.7\% difference in bond length) with a centered $\mathrm{Li}$ ion as the buffer element and shows a $C 2 / m$ monoclinic symmetry [84]. Magnetic susceptibility characterization indicates a Curie-Weiss temperature of $-33 \mathrm{~K}$, with an effective moment of $\mu_{\text {eff }}=1.83(5) \mu_{B}$. A Néel transition at $T_{N} \approx 15 \mathrm{~K}$ is further observed in accordance with specific heat measurements [76]. The resulting antiferromagnetic ground state is of an incommensurate counter-rotating type in the Ir plane [84].

X-ray diffraction studies together with DFT calculations [83] showed a structural phase transition under pressure at $P_{c}=3.8 \mathrm{GPa}$, from the monoclinic $C 2 / \mathrm{m}$ to a dimerized triclinic $P \overline{1}$ structure. With an increasing Z1 bond, the dimerization is taking place either in the X1 or in the Y1 Ir-Ir bond of the honeycomb, resulting in two possible order patterns (cf. Figure $7 b, c)$ in the high-pressure phase. Furthermore, it was elaborated that different properties balance, whether an energy gain by forming a magnetic order or a dimerization is higher. These are the size of the buffer ion and the electronic configuration of the metal species. The latter influences the strength of spin-orbit coupling, electronic correlations, and Hund's rule coupling. Larger interactions (SOC, $U$, and $J_{H}$ ) protect Kitaev physics by ensuring Ising-like spins and by inhibiting dimerization [83]. A larger buffer ion, in principle, inhibits dimerization by an additional hardening of the lattice. Thus, it was argued that $\alpha-\mathrm{Li}_{2} \mathrm{IrO}_{3}$ is the intermediate case between $\mathrm{Na}_{2} \mathrm{IrO}_{3}$ and $\mathrm{Li}_{2} \mathrm{RuO}_{3}$. Indeed, $\mathrm{Li}_{2} \mathrm{RuO}_{3}$ already dimerizes at ambient conditions [85], whereas $\mathrm{Na}_{2} \mathrm{IrO}_{3}$ (discussed below) with a larger center ion size ( $\mathrm{Na}$ vs. $\mathrm{Li}$ ) is expected to dimerize at higher pressure.

Furthermore, studies using multiple X-ray techniques-X-ray powder diffraction (XRD) investigating the crystal structure, resonant inelastic X-ray scattering (RIXS) unveiling the electronic 
structure, and X-ray absorption spectroscopy (XAS) probing SOC under hydrostatic pressure together with DFT calculations-give additional insights [86]. At low pressures of about $0.1 \mathrm{GPa}$, the X-ray powder diffraction shows a gradual elongation of the honeycomb, where two long bonds (3.08 $\AA$ ) and four short bonds $(2.92 \AA)$, still within the $C 2 / \mathrm{m}$ symmetry, are formed. The XAS data suggest a strongly decreasing SOC up to $1.1 \mathrm{GPa}$, saturating at around 2.8 GPa. Interestingly, the RIXS spectra in Figure $7 \mathrm{~d}$ indicate a pressure dependence of the crystal field excitations. At low pressure, the SO-exciton (peak A) with corresponding energy of $\frac{3 \lambda}{2} \approx 0.72 \mathrm{eV}$ is clearly identified. While this excitation should, in principle, be splitted due to reasonable trigonal crystal field splitting $\Delta_{T r} \approx 0.11 \mathrm{eV}$ (see Figure 7e), a substructure is not resolved within the resolution of the setup. Under pressure, the corresponding intensity gets suppressed and peak A slightly shifts to lower energies. A new peak B develops at around $1.4 \mathrm{eV}$ and gets intensified under pressure. This was interpreted as an increase of trigonal crystal field splitting over spin-orbit coupling. Consequently, Clancy et al. argued that the relativistic $j_{e f f}$ picture breaks down, even at low pressures of around $0.1 \mathrm{GPa}$, in favour of a localized pseudospin approach or an itinerant quasimolecular orbital (QMO) model (Figure 7f). At around $3 \mathrm{GPa}$, powder diffraction identified a first-order structural transition in accordance with Reference [83]. Here, a pronounced transfer of spectral weight from peak A to peak B is observed. Fitting of Peak B unveiled a two-peak structure related to the possible transitions to a QMO picture.

Note that there is a pressure-dependent optical study on this compound [87].
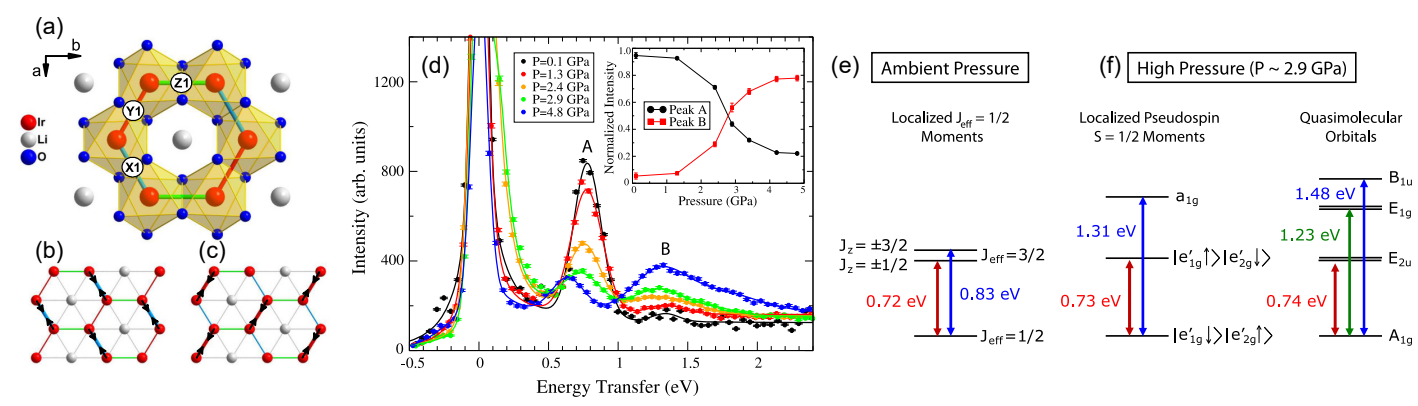

Figure 7. (a) Below $P_{c}$, the honeycomb of $\alpha-\mathrm{Li}_{2} \mathrm{IrO}_{3}$ realizes symmetry-equivalent $\mathrm{X} 1, \mathrm{Y} 1$, and distinct $\mathrm{Z} 1$ bonds. $(\mathbf{b}, \mathbf{c})$ The high-pressure dimerized phase consists of two degenerate order patterns. Reproduced from Reference [83]. (d) Pressure-dependent resonant inelastic X-ray scattering (RIXS) on $\alpha-\mathrm{Li}_{2} \operatorname{IrO}_{3}$ [86]. At ambient pressure, the SO-exciton (peak A) can be clearly identified according to the level structure of the $j_{\text {eff }}$ model (e). At higher pressure (around $1.4 \mathrm{GPa}$ ), a second contribution (peak B) emerges, related to an enhancement of $\Delta_{T r}$. Below $2 \mathrm{GPa}$, the two peaks A and B can be fitted to the transitions of a pseudospin model or an itinerant quasimolecular orbital (QMO) state equivalently well. In the high-pressure phase, peak B shows a two-peak structure with a contribution ar around $1.6 \mathrm{eV}$, interpreted as a transition from the $j_{\text {eff }}$ picture to a QMO state (f).

\subsection{2. $\beta-\mathrm{Li}_{2} \mathrm{IrO}_{3}$}

The $\beta$-polymorph forms a hyper-honeycomb structure of edge-sharing $\mathrm{IrO}_{6}$ octahedra with nearly identical Ir-O-Ir bonds ( $0.2 \%$ difference) and angles of around $94.5^{\circ}$. It extends Kitaev physics in three dimensions [88]. This relativistic Mott insulator has effective moments of $\mu_{\text {eff }}=1.6(1) \mu_{B}$, and the magnetic susceptibility shows a positive Curie-Weiss temperature of $40 \mathrm{~K}$, which may stem from ferromagnetic Kitaev couplings [89]. A transition to a noncollinear or incommensurate ground state at $38 \mathrm{~K}$ is seen under rather strong fluctuations. Furthermore, an unusual cusp in specific heat measurements [88] indicates a second-order transition. The low ferromagnetic Curie-Weiss temperature was interpreted as an effective cancellation of two competitive and nearly degenerate ferromagnetic (possibly Kitaev exchange) and antiferromagnetic ground states. These observations were interpreted as proximity to a Kitaev QSL [88]. Furthermore, Raman spectroscopy indicates signatures of fractionalized excitations [90], similar to $\alpha-\mathrm{RuCl}_{3}$.

A relative weak magnetic field of $3 \mathrm{~T}$ polarizes the compound with $0.35 \mu_{B} / \operatorname{Ir}$ [88] and induces strongly correlated ferromagnetic zigzag chains. Magnetic resonant X-ray scattering [91] shows 
a thermal driven crossover from a paramagnetic behavior in this quantum correlated (quantum paramagnetic) state. The field-induced moments were traced by $\mathrm{X}$-ray magnetic circular dichroism (XMCD) [88] to be suppressed under pressure at $1 \mathrm{GPa}$ and vanished over $2 \mathrm{GPa}$, while the compound remained insulating. Finally, this was interpreted as a rearrangement of the $j_{\text {eff }}$ moments. By applying pressure without external magnetic field, the order temperature shifts first from around $38 \mathrm{~K}$ to $15 \mathrm{~K}$ [88]. Under further increase, $\beta$ - $\mathrm{Li}_{2} \mathrm{IrO}_{3}$ undergoes an electronic/magnetic phase transition at $1.5 \mathrm{GPa}$, as observed by X-ray absorption near edge structure (XANES) measurements [92], without breaking the lattice symmetry. Probing the $5 \mathrm{~d}$ holes, SOC was found to be reduced but remains important. $A b$ initio calculations indicate a dominant Dzyaloshinskii-Moriya regime under pressure, pushed away from the pure $j_{e f f}$ limit. However, the compound still remains in a relativistic Mott picture with an enlarged mixture between $j_{e f f}=3 / 2$ and $j_{\text {eff }}=1 / 2$ states $[88,93]$. The new ground state remains intriguing [94]. Around $4 \mathrm{GPa}$ further, a phase transition to a monoclinic $C 2 / \mathrm{m}$ symmetry was observed [92]. The compound dimerizes under a compression of $X$ and $Y$ Ir-Ir bonds, compared to the $\mathrm{Z}$ bonds.

\subsection{3. $\gamma-\mathrm{Li}_{2} \mathrm{IrO}_{3}$}

The zigzag chains in $\gamma-\mathrm{Li}_{2} \mathrm{IrO}_{3}$ host $j_{e f f}=1 / 2$ spins with $\mu_{e f f}=1.6 \mu_{B}$ in a noncoplanar, counter-rotating pattern $[95,96]$. A transition temperature of about $38 \mathrm{~K}$ and a strongly anisotropic susceptibility, which rather does not allow a determined Curie-Weiss temperature, were observed. The underlying lattice is of $\mathrm{Cccm}$ symmetry. Equally to $\beta-\mathrm{Li}_{2} \mathrm{IrO}_{3}$, Raman spectroscopy shows signatures of fractionalized excitations [90]. Resonant X-ray scattering (RXS) measurements under pressure find an abrupt suppression of the spiral magnetic order at 1.4 GPa without indications of a changed lattice symmetry and point out a continuous reduction of the unit cell volume [97]. This non-magnetic pressure state remains of further interest.

\subsection{4. $\mathrm{Na}_{2} \mathrm{IrO}_{3}$}
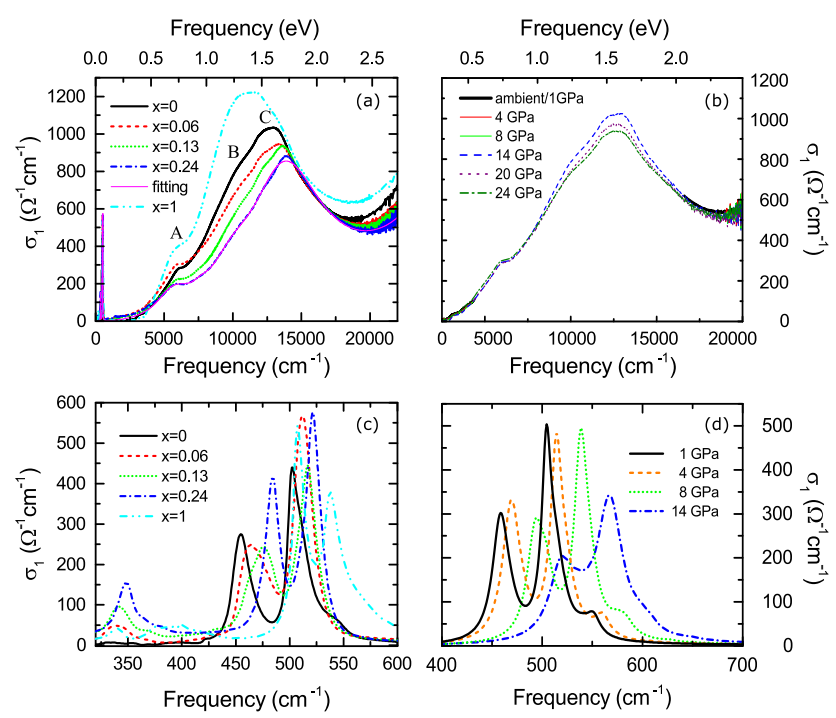

Figure 8. Infrared spectroscopy combined with pressure and isoelectric doping studies on $\mathrm{Na}_{2} \mathrm{IrO}_{3}$ of Reference [98]: The effect of $\mathrm{Li}$ doping $\left(\left(\mathrm{Na}_{1-x} \mathrm{Li}_{x}\right)_{2} \mathrm{IrO}_{3}\right.$ for $x \leq 0.24$ and $x=1$ (represented by $\alpha$ - $\mathrm{Li}_{2} \mathrm{IrO}_{3}$ )) on the electronic spectra (a) and phononic part (c) is shown. (a) While the SO-exciton (peak A) is only marginally affected by Li doping, the direct hopping between $j_{\text {eff }}=1 / 2$ orbitals (peak B) gets suppressed. A blueshift of the intersite $j_{\text {eff }}=3 / 2 \rightarrow j_{\text {eff }}=1 / 2$ transition (peak C) for $x \leq 0.24 \mathrm{Li}$ doping indicates a increasing $U_{e f f} / t$. (c) The phononic part shows a hardening upon increasing the Li doping. Further, the effect of hydrostatic pressure on the electronic spectra (b) and on the phononic part (d) is shown. (b) Under pressure $U_{e f f} / t$ is slightly lowered, indicated by a redshift of all features while the lattice is contracted (d). 
The layered honeycomb of $\mathrm{Na}_{2} \mathrm{IrO}_{3}$ is similar to $\alpha-\mathrm{Li}_{2} \mathrm{IrO}_{3}$, with edge-sharing $\mathrm{IrO}_{6}$ octahedra and $\mathrm{Na}$ as buffer ion (C2/m space group). Ir-O-Ir bond angles reach from $98^{\circ}$ to $99.4^{\circ}$ [99]. High-temperature moments of $\mu_{e f f}=1.79 \mu_{B}$ are determined together with a Curie-Weiss temperature of $-125 \mathrm{~K}$ [76]. Below 15-18 K, an antiferromagnetic zigzag order is observed with 0.22(1) $\mu_{B} / \operatorname{Ir}[99,100]$. Spin-wave excitations in this magnetic order were studied by inelastic neutron scattering experiments and compared with theoretical considerations [99], showing the importance of higher-order coupling contributions on the honeycomb lattice [101].

Optical studies established a mostly temperature-independent onset of a Mott gap at around $340 \mathrm{meV}$ [102]. Electronic features below $3 \mathrm{eV}$ are assigned to be transitions, belonging to the $\operatorname{Ir} 5 \mathrm{~d} t_{2 g}$ multiplets, and above mostly to charge transfer transition from O $2 \mathrm{p}$ to $\operatorname{Ir} 5 \mathrm{~d} t_{2 g}$. A clear absorption edge is visible, resulting in an effective on-site Coulomb repulsion of about $1.5 \mathrm{eV}$. This seems to match the LDA+SOC $+U$ calculated DOS with $U=3 \mathrm{eV}$ and $J_{H}=0.6 \mathrm{eV}$ [102]. The $j_{\text {eff }}$ picture is clearly valid as pointed out by resonant inelastic $\mathrm{X}$-ray scattering. These studies show a splitted but pronounced SO-exciton, concluding that trigonal distortions are weaker compared to a strong SOC $(110 \mathrm{meV}$ and $0.4-0.5 \mathrm{eV}$, respectively) [78].

Hydrostatic pressure and isoelectric doping studies on $\mathrm{Na}_{2} \mathrm{IrO}_{3}$ using infrared spectroscopy and synchrotron X-ray diffraction were performed by Hermann et al. [98]. The optical conductivity under ambient pressure for an as-grown sample is displayed in Figure 8a (black line). The $d-d$ contributions peak $\mathrm{A}(0.7 \mathrm{eV})$, peak $\mathrm{B}(1.2 \mathrm{eV})$, and peak $\mathrm{C}(1.6 \mathrm{eV})$ (Figure $8 \mathrm{a})$ are assigned as follows: intrasite $j_{e f f}=3 / 2 \rightarrow j_{e f f}=1 / 2$, intersite $j_{\text {eff }}=1 / 2 \rightarrow j_{\text {eff }}=1 / 2$, and intersite $j_{\text {eff }}=3 / 2 \rightarrow j_{\text {eff }}=1 / 2$ transitions, respectively, and thus probing the Mott insulating picture directly (peak A) and Kitaev correlations indirectly (peak B and C). The first intersite excitation peak B reveals a Mott gap with $U_{\text {eff }}=1.2 \mathrm{eV}$, in accordance with Reference [102]. On the low-energy side, phononic excitations contribute (Figure $8 \mathrm{c}$ ). While the $\mathrm{C} 2 / \mathrm{m}$ symmetry hosts 18 infrared-active modes, five were resolved by the experiment in the undoped sample at ambient pressure.

The effect of Li doping, discriminating $\mathrm{Na}$, with $\left(\mathrm{Na}_{1-x} \mathrm{Li}_{x}\right)_{2} \mathrm{IrO}_{3}$ for $x \leq 0.24$ and $x=1$ (represented by $\alpha-\mathrm{Li}_{2} \mathrm{IrO}_{3}$ ) is analyzed in detail. Focusing on the electronic part (Figure 8a), the intrasite contribution (peak A) remains mostly stable upon doping, with only a slight redshift, while the intersite excitation (peak C) shifts to higher energies. This indicates an increasing ratio of $U_{e f f} / t$, while SOC and a distortion of the crystal field remain only marginally affected. Further, it corresponds to a shift towards the Mott insulating side. According to theoretical predictions [103], the decreasing spectral weight of the $j_{e f f}=1 / 2 \rightarrow j_{\text {eff }}=1 / 2$ intersite transition B with increasing amount of doping was related to enhanced Kitaev couplings due to a suppression of the direct Ir-Ir hopping channel. This emphasizes a proximity of the $x=0.24$ compound to the Kitaev limit. Further X-ray measurements show that the chemical pressure upon Li doping only affects a contraction of the $a b$-plane. The $c$ direction remains nearly constant because only in-plane Na sites are affected for a sufficient low doping concentration. This naturally tunes the Ir-O-Ir bond angle, crucially influencing Kitaev magnetism. However, $\alpha-\mathrm{Li}_{2} \mathrm{IrO}_{3}$ is located not so deep in the Mott insulating state, as indicated by a redshifted absorption edge (Figure 8a), therefore resulting in a lower ratio of $U_{e f f} / t$, backed by previous studies [60]. The similar position and shape of transition A prove a comparable SOC and distortion of the crystal field in $\mathrm{Na}_{2} \mathrm{IrO}_{3}$ and $\alpha-\mathrm{Li}_{2} \mathrm{IrO}_{3}$ [55].

On the phononic part (Figure $8 \mathrm{c}$ ), increasing the Li doping is expressed as a hardening of the in-plane modes due to compression of the $a b$-plane. In addition to this chemical pressure effect, the phonon modes are intrinsically affected by the contribution of Li. The observed modes, previously without any Na contribution, were simulated to have an increasing contribution of Li discriminating Ir. The lowest mode at around $350 \mathrm{~cm}^{-1}$ was found to be purely Li based, in accordance to the optical spectra.

Hermann et al. further compared the effect of chemical pressure to the physical hydrostatic one. The optical conductivity for pressurized samples is shown in Figure 8b,d. On the electronic part (Figure $8 \mathrm{~b}$ ), hydrostatic pressure over $8 \mathrm{GPa}$ leads to a decreasing intensity of the absorption 
edge, while the intrasite transition (peak A) remains nearly unaffected. A slight redshift of all features upon increasing pressure is observed. $U_{e f f} / t$ is therefore only slightly adjusted between $8 \mathrm{GPa}$ and $24 \mathrm{GPa}$. The nearly unchanged intrasite contribution indicates a smooth monotonic contraction without disturbing the crystal field symmetry. In addition, the phononic contributions (Figure 8d) were found to experience a monotonic hardening while the damping increases with pressure. Overall, no indications for a breaking of lattice symmetry are found. Further X-ray measurements show that, additionally to the $a b$-plane, the $c$ direction is contracted upon pressurizing. This naturally explains the different affected intensities of peak B and peak $C$. While transition $C j_{\text {eff }}=3 / 2 \rightarrow j_{\text {eff }}=1 / 2$ is influenced by changes of the Ir-O-Ir bond angle, the direct hopping of peak B is nearly unchanged through the smooth contraction of the $a b$-plane. Thus, it was argued that external hydrostatic pressure, in contrast to the chemical counterpart, drives the compound away from the Kitaev limit.

\section{Kagome Lattice}

(a)

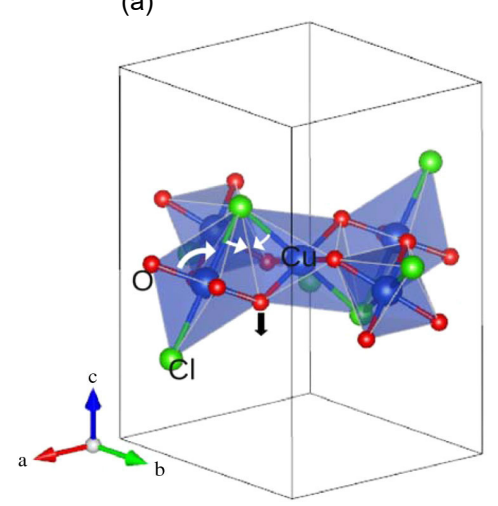

(b)

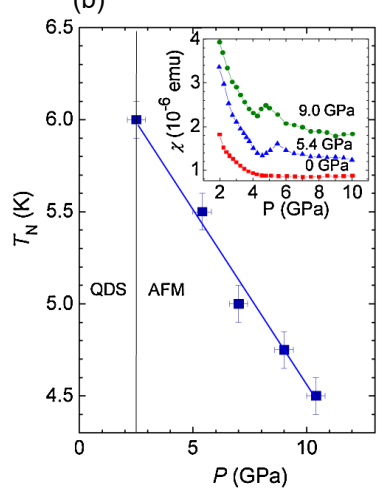

(c)

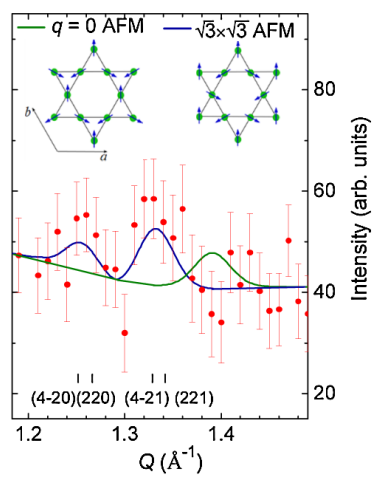

Figure 9. (a) Pressure effect on the crystal structure of Herbertsmithite. While the $R \overline{3} m$ symmetry is preserved under pressure, trigonal distortion is added by tilting the $\mathrm{CuO}_{4}$-plane. (b) Phase diagram under pressure: At 2.1 GPa, a transition from a QSL to an AFM ordered phase takes place, which can be seen in the susceptibility data (inset). (c) Neutron diffraction pattern under pressure. The local spin structure under pressure resembles a $\sqrt{3} \times \sqrt{3}$ type. After Reference [104].

Herbertsmithite $\mathrm{ZnCu}_{3}(\mathrm{OH})_{6} \mathrm{Cl}_{2}$ is one of the most highlighted QSL model systems, crystallizing in the highly frustrated Kagome lattice (see Reference [105] and references herein). Cu Kagome planes $(S=1 / 2)$ are separated by $\mathrm{Zn}^{2+}$ ions realizing a highly frustrated system. Note that there is a prone to disorder induced by mixing of $\mathrm{Cu}$ and $\mathrm{Zn}$ ions [106]. Besides strong antiferromagnetic interaction $(J=190 \mathrm{~K})$ due to the $120^{\circ} \mathrm{Cu}$ bonds, no signature of magnetic order was found down to the lowest temperatures of around $50 \mathrm{mK}$ [107,108]. Furthermore, Dzyaloshinskii-Moriya interaction was found to be important [109]. Spin freezing was observed under magnetic field [110] and pressure [104], on which we want to discuss now in detail. Under pressure, the $R \overline{3} m$ symmetry is preserved up to at least $5 \mathrm{GPa}$. However, $\mathrm{Cu}-\mathrm{O}-\mathrm{Cu}$ bond angles are non-monotonically affected. First, for $P<0.25 \mathrm{GPa}$, a linear increase is observed, followed by a decrease up to $5.1 \mathrm{GPa}$. The $\mathrm{Cu}-\mathrm{O}$ bond distance was found to decrease linearly at the low pressure side of $P<0.25 \mathrm{GPa}$ and to be pressure independent above. Figure 9a shows the pressure effect on the crystal structure of Herbertsmithite. Most importantly, the $\mathrm{CuO}_{4}$-plane tilts respectively to the $\mathrm{Cl}-\mathrm{Cu}-\mathrm{Cl}$ axis, inducing a trigonal distortion. Interestingly, the ratio of DM and Heisenberg interaction in Herbertsmithite is lowered under pressure. A quantum phase transition from the QSL ground state to an ordered AFM phase is observed at $2.5 \mathrm{GPa}$ with $T_{N}=6 \mathrm{~K}$ as a peak in susceptibility measurements (Figure $9 \mathrm{~b}$ ). In the AFM ordered state, $T_{N}$ is further decreased under pressure and explained by a decrease of Heisenberg interaction (15\% from 2.5 GPa to $5.1 \mathrm{GPa}$ ). The AFM structure is of type $\sqrt{3} \times \sqrt{3}$ (see Figure 9c). 


\section{Spin 1/2 Dimer Systems}

We also want to highlight pressure-dependent studies on the spin dimer system $\mathrm{TlCuCl}_{3}[111,112]$. This magnetic insulator host dimerized $S=1 / 2$ moments of $\mathrm{Cu}^{2+}$ ions confined by strong AFM interaction. The formation of spin dimers leads to a quantum disordered phase at ambient pressure. Between the singlet ground state $(S=0)$ and the first excited triplet state $(S=1)$, there is a small gap of about $0.7 \mathrm{meV}$ for spin excitations. Pressure [113-117], magnetic field [118], and impurity doping were shown to generate AFM order [119]. We are going to review the pressure-induced phase transition in detail. In a simple picture, the interdimer coupling can be increased by external pressure, closing the spin gap [120]. Rüegg et al. found a quantum phase transition (QCP) at $P=0.107 \mathrm{GPa}$ and a power law increase of $T_{N}$ in the AFM phase [116]. Spin dimer formation can be destroyed by the suppression of quantum fluctuations or the reduction of thermal fluctuations, both leading to magnetic order. Figure 10 summarizes both phase transitions as probed by inelastic neutron scattering [117]. First, we focus on the QPT (Figure 10a). As the pressure is increased, the spin gap is suppressed and finally closed with a temperature-dependent pressure $p_{c}$. In the ordered Néel state (right-hand side), two types of excitations are observed, namely the conventional Goldstone mode or spin wave (Figure 10a grey symbols) and, remarkably, the longitudinal Higgs mode (Figure 10a red symbols) [121]. Decreasing the pressure softens the Higgs mode, and finally as dimer-based quantum fluctuations destroy magnetic order, the system becomes gapped under $p_{c}$. While, transverse excitation remains gapped $\left(0.38 \mathrm{meV}\right.$ at $\left.p_{c}\right)$ over the full pressure range, they can be well distinguished from the gapless longitudinal modes at $p_{c}$. Now, we want to focus on the classical phase transition (Figure 10b). At high temperatures, thermal fluctuations gap all observed modes. Having the temperature as a tuning parameter and a fixed pressure of $0.175 \mathrm{GPa}$, the ordered state emerges below $T_{N}$ as the longitudinal mode becomes gapless at $T_{N}$. Here, we see that quantum and thermal melting of the ordered phase are affecting the neutron spectra in a qualitatively very similar way. Finally, a full phase diagram covering quantum critical and classical critical region is shown in Figure 10c.
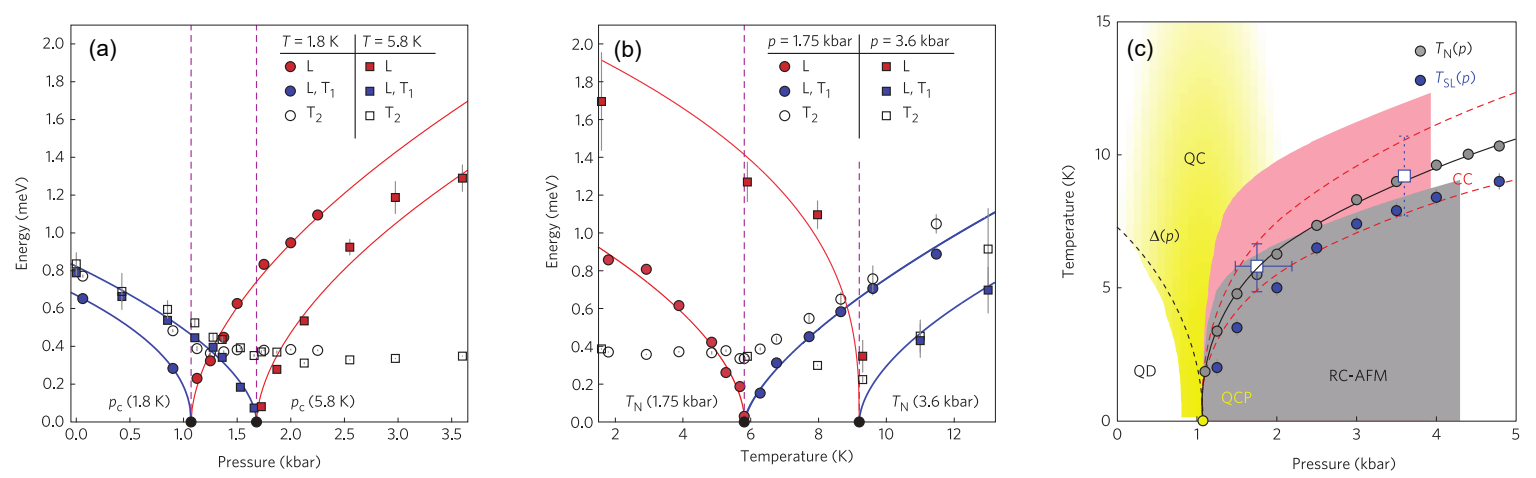

Figure 10. Quantum and classical phase transition from a dimer $S=1 / 2$ state to magnetic order in $\mathrm{TlCuCl}_{3}$ observed by inelastic neutron scattering: $(\mathbf{a}, \mathbf{b})$ Evolution of mode energies for pressure and temperature as tuning parameters. At the quantum critical point, QPT takes place: (a) Transverse magnetic modes $(\mathrm{T})$ or Goldstone mode of the ordered phase remains gapped while the longitudinal Higgs mode (L) is gapless at the QCP with a temperature-dependent $p_{c}$. Taking temperature as the tuning parameter $(\mathbf{b})$ and a fixed pressure, the ordered state emerges at a pressure-dependent $T_{N}$. A qualitatively similar evolution of the mode gaps for classical and quantum phase transition is observed. The results are summarized in the phase diagram (c) showing the quantum disordered state (QD) and induced magnetic phase (RC-AFM). Quantum critical and classical critical regions are indicated as QC, and CC, respectively. Grey spheres show the power-law behavior of $T_{N}(p)$, while blues symbols $\left(T_{S L}(p)\right)$ denote the limit of classical scaling. Reproduced from Reference [117]. 


\section{Summary}

In summary, external pressure can be a very powerful tool to tune the electronic, magnetic, and structural parameters opening a new route in the investigations of QSLs and candidates. For instance, small perturbations to the crystal structure can drive the geometrical frustration factor towards a favourable state. More complicated but possible is a direct tuning of the exchange coupling $\left(\mathrm{Cs}_{2} \mathrm{CuCl}_{4}\right)$. Moreover, unwanted magnetic interactions and fluctuations can be suppressed, leaving room for realization of a pure QSL state. However, it is often difficult to predict the influence of external pressure on magnetic properties of a candidate system. In fact, we have seen few systems where the pressure induces spin freezing rather than a liquid state (for instance, in $\mathrm{Tb}_{2} \mathrm{Ti}_{2} \mathrm{O}_{7}$ or $\mathrm{Yb}_{2} \mathrm{Ti}_{2} \mathrm{O}_{7}$ ) or an unfavourable modification of the crystal structure $\left(\alpha-\mathrm{RuCl}_{3}\right)$. Promising are candidates where a magnetic order vanishes before the structural transition as in the case of the iridate systems. Therefore, there is no easy answer for whether external pressure is always a pathway to introduce spin liquid physics in the candidate systems. Albeit that the end results are unpredictable, exotic states of matter in the vicinity of the QSL state can be investigated.

Author Contributions: T.B. wrote the manuscript. T.B. and E.U. discussed the content. All authors have read and agreed to the published version of the manuscript.

Funding: This research was funded by Deutsche Forschungsgemeinschaft through grant No. DR228/52-1. and the "Margarete von Wrangell Habilitation Programm" by the Ministry of Sciences, Research, and Arts in Baden Württemberg.

Acknowledgments: We thank Weiwu Li, Seulki Roh, Andrej Pustogow, Artem Pronin, and Martin Dressel for fruitful discussions. T.B. acknowledges funding from Deutsche Forschungsgemeinschaft through grant No. DR228/52-1. E.U. acknowledges the support by “Margarete von Wrangell Habilitation Programm” by the Ministry of Sciences, Research, and Arts in Baden Württemberg.

Conflicts of Interest: The authors declare no conflict of interest.

\section{References}

1. Pesin, D.; Balents, L. Mott physics and band topology in materials with strong spin-orbit interaction. Nat. Phys. 2010, 6, 376-381. [CrossRef]

2. Wen, X.G. Colloquium: Zoo of quantum-topological phases of matter. Rev. Mod. Phys. 2017, 89, 041004. [CrossRef]

3. Anderson, P.W. Resonating valence bonds: A new kind of insulator? Mater. Res. Bull. 1973, 8, $153-160$. [CrossRef]

4. Kitaev, A. Anyons in an exactly solved model and beyond. Ann. Phys. 2006, 321, 2-111. [CrossRef]

5. Nasu, J.; Udagawa, M.; Motome, Y. Vaporization of Kitaev Spin Liquids. Phys. Rev. Lett. 2014, $113,197205$. [CrossRef]

6. Savary, L.; Balents, L. Quantum spin liquids: A review. Rep. Prog. Phys. 2016, 80, 016502. [CrossRef]

7. Zhou, Y.; Kanoda, K.; Ng, T.K. Quantum spin liquid states. Rev. Mod. Phys. 2017, 89, 025003. [CrossRef]

8. Hermanns, M.; Kimchi, I.; Knolle, J. Physics of the Kitaev Model: Fractionalization, Dynamic Correlations, and Material Connections. Annu. Rev. Condens. Matter Phys. 2018, 9, 17-33. [CrossRef]

9. Takagi, H.; Takayama, T.; Jackeli, G.; Khaliullin, G.; Nagler, S.E. Concept and realization of Kitaev quantum spin liquids. Nat. Rev. Phys. 2019, 1, 264-280. [CrossRef]

10. Knolle, J.; Moessner, R. A Field Guide to Spin Liquids. Annu. Rev. Condens. Matter Phys. 2019, 10, 451-472. [CrossRef]

11. Wen, J.; Yu, S.L.; Li, S.; Yu, W.; Li, J.X. Experimental identification of quantum spin liquids. Npj Quantum Mater. 2019, 4, 12. [CrossRef]

12. Anderson, P.W. The Resonating Valence Bond State in $\mathrm{La}_{2} \mathrm{CuO}_{4}$ and Superconductivity. Science 1987, 235, 1196. [CrossRef] [PubMed]

13. Powell, B.J.; McKenzie, R.H. Quantum frustration in organic Mott insulators: From spin liquids to unconventional superconductors. Rep. Prog. Phys. 2011, 74, 056501. [CrossRef]

14. Dressel, M.; Pustogow, A. Electrodynamics of quantum spin liquids. J. Phys. Condens. Matter 2018, 30, 203001. [CrossRef] [PubMed] 
15. Morris, D.J.P.; Tennant, D.A.; Grigera, S.A.; Klemke, B.; Castelnovo, C.; Moessner, R.; Czternasty, C.; Meoptissner, M.; Rule, K.C.; Hoffmann, J.U.; et al. Dirac Strings and Magnetic Monopoles in the Spin Ice $\mathrm{Dy}_{2} \mathrm{Ti}_{2} \mathrm{O}_{7}$. Science 2009, 326, 411-414. [CrossRef] [PubMed]

16. Bramwell, S.T.; Gingras, M.J.P. Spin Ice State in Frustrated Magnetic Pyrochlore Materials. Science 2001, 294, 1495-1501. [CrossRef] [PubMed]

17. Kermarrec, E.; Gaudet, J.; Fritsch, K.; Khasanov, R.; Guguchia, Z.; Ritter, C.; Ross, K.A.; Dabkowska, H.A.; Gaulin, B.D. Ground state selection under pressure in the quantum pyrochlore magnet $\mathrm{Yb}_{2} \mathrm{Ti}_{2} \mathrm{O}_{7}$. Nat. Commun. 2017, 8, 14810. [CrossRef]

18. Mishra, A.K.; Poswal, H.K.; Sharma, S.M.; Saha, S.; Muthu, D.V.S.; Singh, S.; Suryanarayanan, R.; Revcolevschi, A.; Sood, A.K. The study of pressure induced structural phase transition in spin-frustrated $\mathrm{Yb}_{2} \mathrm{Ti}_{2} \mathrm{O}_{7}$ pyrochlore. J. Appl. Phys. 2012, 111, 033509. [CrossRef]

19. Dun, Z.L.; Lee, M.; Choi, E.S.; Hallas, A.M.; Wiebe, C.R.; Gardner, J.S.; Arrighi, E.; Freitas, R.S.; Arevalo-Lopez, A.M.; Attfield, J.P.; et al. Chemical pressure effects on magnetism in the quantum spin liquid candidates $\mathrm{Yb}_{2} \mathrm{X}_{2} \mathrm{O}_{7}(\mathrm{X}=\mathrm{Sn}, \mathrm{Ti}, \mathrm{Ge})$. Phys. Rev. B 2014, 89, 064401. [CrossRef]

20. Rittman, D.R.; Turner, K.M.; Park, S.; Fuentes, A.F.; Yan, J.; Ewing, R.C.; Mao, W.L. High-pressure behavior of $\mathrm{A}_{2} \mathrm{~B}_{2} \mathrm{O}_{7}$ pyrochlore (A=Eu, Dy; $\mathrm{B}=\mathrm{Ti}, \mathrm{Zr}$ ). J. Appl. Phys. 2017, 121, 045902. [CrossRef]

21. Mirebeau, I.; Goncharenko, I.N.; Dhalenne, G.; Revcolevschi, A. Pressure and Field Induced Magnetic Order in the Spin Liquid $\mathrm{Tb}_{2} \mathrm{Ti}_{2} \mathrm{O}_{7}$ as Studied by Single Crystal Neutron Diffraction. Phys. Rev. Lett. 2004, 93, 187204. [CrossRef] [PubMed]

22. Gardner, J.S.; Dunsiger, S.R.; Gaulin, B.D.; Gingras, M.J.P.; Greedan, J.E.; Kiefl, R.F.; Lumsden, M.D.; MacFarlane, W.A.; Raju, N.P.; Sonier, J.E.; et al. Cooperative Paramagnetism in the Geometrically Frustrated Pyrochlore Antiferromagnet $\mathrm{Tb}_{2} \mathrm{Ti}_{2} \mathrm{O}_{7}$. Phys. Rev. Lett. 1999, 82, 1012-1015. [CrossRef]

23. Gingras, M.; den Hertog, B.; Faucher, M.; Gardner, J.; Dunsiger, S.; Chang, L.; Gaulin, B.; Raju, N.; Greedan, J. Thermodynamic and single-ion properties of $\mathrm{Tb}^{3+}$ within the collective paramagnetic-spin liquid state of the frustrated pyrochlore antiferromagnet $\mathrm{Tb}_{2} \mathrm{Ti}_{2} \mathrm{O}_{7}$. Phys. Rev. B 2000, 62, 6496-6511. [CrossRef]

24. Gardner, J.S.; Keren, A.; Ehlers, G.; Stock, C.; Segal, E.; Roper, J.M.; Fåk, B.; Stone, M.B.; Hammar, P.R.; Reich, D.H.; et al. Dynamic frustrated magnetism in $\mathrm{Tb}_{2} \mathrm{Ti}_{2} \mathrm{O}_{7}$ at $50 \mathrm{mK}$. Phys. Rev. B 2003, 68, 180401. [CrossRef]

25. Lhotel, E.; Paulsen, C.; de Réotier, P.D.; Yaouanc, A.; Marin, C.; Vanishri, S. Low-temperature magnetization in geometrically frustrated $\mathrm{Tb}_{2} \mathrm{Ti}_{2} \mathrm{O}_{7}$. Phys. Rev. B 2012, 86, 020410. [CrossRef]

26. Legl, S.; Krey, C.; Dunsiger, S.R.; Dabkowska, H.A.; Rodriguez, J.A.; Luke, G.M.; Pfleiderer, C. Vibrating-Coil Magnetometry of the Spin Liquid Properties of $\mathrm{Tb}_{2} \mathrm{Ti}_{2} \mathrm{O}_{7}$. Phys. Rev. Lett. 2012, 109, 047201. [CrossRef]

27. Hallas, A.M.; Cheng, J.G.; Arevalo-Lopez, A.M.; Silverstein, H.J.; Su, Y.; Sarte, P.M.; Zhou, H.D.; Choi, E.S.; Attfield, J.P.; Luke, G.M.; et al. Incipient Ferromagnetism in $\mathrm{Tb}_{2} \mathrm{Ge}_{2} \mathrm{O}_{7}$ : Application of Chemical Pressure to the Enigmatic Spin-Liquid Compound $\mathrm{Tb}_{2} \mathrm{Ti}_{2} \mathrm{O}_{7}$. Phys. Rev. Lett. 2014, 113, 267205. [CrossRef]

28. Molavian, H.R.; Gingras, M.J.P.; Canals, B. Dynamically Induced Frustration as a Route to a Quantum Spin Ice State in $\mathrm{Tb}_{2} \mathrm{Ti}_{2} \mathrm{O}_{7}$ via Virtual Crystal Field Excitations and Quantum Many-Body Effects. Phys. Rev. Lett. 2007, 98, 157204. [CrossRef]

29. Bonville, P.; Mirebeau, I.; Gukasov, A.; Petit, S.; Robert, J. Tetragonal distortion yielding a two-singlet spin liquid in pyrochlore $\mathrm{Tb}_{2} \mathrm{Ti}_{2} \mathrm{O}_{7}$. Phys. Rev. B 2011, 84, 184409. [CrossRef]

30. Fennell, T.; Kenzelmann, M.; Roessli, B.; Mutka, H.; Ollivier, J.; Ruminy, M.; Stuhr, U.; Zaharko, O.; Bovo, L.; Cervellino, A.; et al. Magnetoelastic Excitations in the Pyrochlore Spin Liquid $\mathrm{Tb}_{2} \mathrm{Ti}_{2} \mathrm{O}_{7}$. Phys. Rev. Lett. 2014, 112, 017203. [CrossRef]

31. Mirebeau, I.; Goncharenko, I.N.; Cadavez-Peres, P.; Bramwell, S.T.; Gingras, M.J.P.; Gardner, J.S. Pressure-induced crystallization of a spin liquid. Nature 2002, 420, 54-57. [CrossRef] [PubMed]

32. Mirebeau, I.; Goncharenko, I.N. $\mathrm{Tb}_{2} \mathrm{Ti}_{2} \mathrm{O}_{7}$ : A 'spin liquid' single crystal studied under high pressure and high magnetic field. J. Phys. Condens. Matter 2005, 17, S771-S782. [CrossRef]

33. Mirebeau, I.; Apetrei, A.; Rodríguez-Carvajal, J.; Bonville, P.; Forget, A.; Colson, D.; Glazkov, V.; Sanchez, J.P.; Isnard, O.; Suard, E. Ordered Spin Ice State and Magnetic Fluctuations in $\mathrm{Tb}_{2} \mathrm{Sn}_{2} \mathrm{O}_{7}$. Phys. Rev. Lett. 2005, 94, 246402. [CrossRef]

34. Kumar, R.S.; Cornelius, A.L.; Somayazulu, M.; Errandonea, D.; Nicol, M.F.; Gardner, J. High pressure structure of $\mathrm{Tb}_{2} \mathrm{Ti}_{2} \mathrm{O}_{7}$ pyrochlore at cryogenic temperatures. Phys. Status Solidi B 2007, 244, 266-269. [CrossRef] 
35. Matsuhira, K.; Hinatsu, Y.; Tenya, K.; Amitsuka, H.; Sakakibara, T. Low-Temperature Magnetic Properties of Pyrochlore Stannates. J. Phys. Soc. Jpn. 2002, 71, 1576-1582. [CrossRef]

36. Sipos, B.; Kusmartseva, A.F.; Akrap, A.; Berger, H.; Forró, L.; Tutiš, E. From Mott state to superconductivity in 1T-TaS 2 . Nat. Mater. 2008, 7, 960-965. [CrossRef]

37. Novoselov, K.S.; Mishchenko, A.; Carvalho, A.; Castro Neto, A.H. 2D materials and van der Waals heterostructures. Science 2016, 353, aac9439. [CrossRef]

38. Law, K.T.; Lee, P.A. 1T-TaS 2 as a quantum spin liquid. Proc. Natl. Acad. Sci. USA 2017, 114, 6996-7000. [CrossRef]

39. Han, T.R.T.; Zhou, F.; Malliakas, C.D.; Duxbury, P.M.; Mahanti, S.D.; Kanatzidis, M.G.; Ruan, C.Y. Exploration of metastability and hidden phases in correlated electron crystals visualized by femtosecond optical doping and electron crystallography. Sci. Adv. 2015, 1, e1400173. [CrossRef]

40. Liu, Y.; Ang, R.; Lu, W.J.; Song, W.H.; Li, L.J.; Sun, Y.P. Superconductivity induced by Se-doping in layered charge-density-wave system 1T-TaS ${ }_{2-x} \mathrm{Se}_{x}$. Appl. Phys. Lett. 2013, 102, 192602. [CrossRef]

41. Gao, J.; Park, J.W.; Kim, K.; Song, S.K.; Chen, F.; Luo, X.; Sun, Y.; Yeom, H.W. Pseudogap and weak multifractality in disordered Mott charge-density-wave insulator. arXiv 2019, arXiv:1904.04508.

42. Lahoud, E.; Meetei, O.N.; Chaska, K.B.; Kanigel, A.; Trivedi, N. Emergence of a Novel Pseudogap Metallic State in a Disordered 2D Mott Insulator. Phys. Rev. Lett. 2014, 112, 206402. [CrossRef]

43. Ritschel, T.; Trinckauf, J.; Garbarino, G.; Hanfland, M.; v. Zimmermann, M.; Berger, H.; Büchner, B.; Geck, J. Pressure dependence of the charge density wave in $1 \mathrm{~T}-\mathrm{TaS}_{2}$ and its relation to superconductivity. Phys. Rev. B 2013, 87, 125135. [CrossRef]

44. Zvyagin, S.A.; Graf, D.; Sakurai, T.; Kimura, S.; Nojiri, H.; Wosnitza, J.; Ohta, H.; Ono, T.; Tanaka, $\mathrm{H}$. Pressure-tuning the quantum spin Hamiltonian of the triangular lattice antiferromagnet $\mathrm{Cs}_{2} \mathrm{CuCl}_{4}$. Nat. Commun. 2019, 10, 1064. [CrossRef] [PubMed]

45. Balents, L. Spin liquids in frustrated magnets. Nature 2010, 464, 199-208. [CrossRef] [PubMed]

46. Zvyagin, S.A.; Kamenskyi, D.; Ozerov, M.; Wosnitza, J.; Ikeda, M.; Fujita, T.; Hagiwara, M.; Smirnov, A.I.; Soldatov, T.A.; Shapiro, A.Y.; et al. Direct Determination of Exchange Parameters in $\mathrm{Cs}_{2} \mathrm{CuBr}_{4}$ and $\mathrm{Cs}_{2} \mathrm{CuCl}_{4}$ : High-Field Electron-Spin-Resonance Studies. Phys. Rev. Lett. 2014, 112, 077206. [CrossRef] [PubMed]

47. Coldea, R.; Tennant, D.A.; Habicht, K.; Smeibidl, P.; Wolters, C.; Tylczynski, Z. Direct Measurement of the Spin Hamiltonian and Observation of Condensation of Magnons in the 2D Frustrated Quantum Magnet $\mathrm{Cs}_{2} \mathrm{CuCl}_{4}$. Phys. Rev. Lett. 2002, 88, 137203. [CrossRef] [PubMed]

48. Starykh, O.A.; Katsura, H.; Balents, L. Extreme sensitivity of a frustrated quantum magnet: $\mathrm{Cs}_{2} \mathrm{CuCl}_{4}$. Phys. Rev. B 2010, 82, 014421. [CrossRef]

49. Coldea, R.; Tennant, D.; Cowley, R.; McMorrow, D.; Dorner, B.; Tylczynski, Z. Neutron scattering study of the magnetic structure of $\mathrm{Cs}_{2} \mathrm{CuCl}_{4}$. J. Phys. Condens. Matter 1996, 8, 7473-7491. [CrossRef]

50. Li, Y.; Chen, G.; Tong, W.; Pi, L.; Liu, J.; Yang, Z.; Wang, X.; Zhang, Q. Rare-Earth Triangular Lattice Spin Liquid: A Single-Crystal Study of $\mathrm{YbMgGaO}_{4}$. Phys. Rev. Lett. 2015, 115, 167203. [CrossRef]

51. Li, Y.; Adroja, D.; Biswas, P.K.; Baker, P.J.; Zhang, Q.; Liu, J.; Tsirlin, A.A.; Gegenwart, P.; Zhang, Q. Muon Spin Relaxation Evidence for the U(1) Quantum Spin-Liquid Ground State in the Triangular Antiferromagnet $\mathrm{YbMgGaO}_{4}$. Phys. Rev. Lett. 2016, 117, 097201. [CrossRef]

52. Majumder, M.; Simutis, G.; Collings, I.E.; Orain, J.C.; Dey, T.; Li, Y.; Gegenwart, P.; Tsirlin, A.A. Persistent spin dynamics in the pressurized spin-liquid candidate $\mathrm{YbMgGaO}_{4}$. arXiv 2019, arXiv:1902.07749.

53. Jackeli, G.; Khaliullin, G. Mott Insulators in the Strong Spin-Orbit Coupling Limit: From Heisenberg to a Quantum Compass and Kitaev Models. Phys. Rev. Lett. 2009, 102, 017205. [CrossRef]

54. Trebst, S. Kitaev Materials. arXiv 2017, arXiv:1701.07056.

55. Winter, S.M.; Tsirlin, A.A.; Daghofer, M.; van den Brink, J.; Singh, Y.; Gegenwart, P.; Valentí, R. Models and materials for generalized Kitaev magnetism. J. Phys. Condens. Matter 2017, 29, 493002. [CrossRef]

56. Banerjee, A.; Lampen-Kelley, P.; Knolle, J.; Balz, C.; Aczel, A.A.; Winn, B.; Liu, Y.; Pajerowski, D.; Yan, J.; Bridges, C.A.; et al. Excitations in the field-induced quantum spin liquid state of $\alpha-\mathrm{RuCl}_{3}$. Npj Quantum Mater. 2018, 3, 8. [CrossRef]

57. Do, S.H.; Park, S.Y.; Yoshitake, J.; Nasu, J.; Motome, Y.; Kwon, Y.S.; Adroja, D.T.; Voneshen, D.J.; Kim, K.; Jang, T.H.; et al. Majorana fermions in the Kitaev quantum spin system $\alpha-\mathrm{RuCl}_{3}$. Nat. Phys. 2017, 13, 1079-1084. [CrossRef] 
58. Plumb, K.W.; Clancy, J.P.; Sandilands, L.J.; Shankar, V.V.; Hu, Y.F.; Burch, K.S.; Kee, H.Y.; Kim, Y.J. $\alpha$-RuCl ${ }_{3}$ : A spin-orbit assisted Mott insulator on a honeycomb lattice. Phys. Rev. B 2014, 90, 041112. [CrossRef]

59. Johnson, R.D.; Williams, S.C.; Haghighirad, A.A.; Singleton, J.; Zapf, V.; Manuel, P.; Mazin, I.I.; Li, Y.; Jeschke, H.O.; Valentí, R.; et al. Monoclinic crystal structure of $\alpha-\mathrm{RuCl}_{3}$ and the zigzag antiferromagnetic ground state. Phys. Rev. B 2015, 92, 235119. [CrossRef]

60. Winter, S.M.; Li, Y.; Jeschke, H.O.; Valentí, R. Challenges in design of Kitaev materials: Magnetic interactions from competing energy scales. Phys. Rev. B 2016, 93, 214431. [CrossRef]

61. Kim, H.S.; Catuneanu, A.; Kee, H.Y. Kitaev magnetism in honeycomb RuCl3 with intermediate spin-orbit coupling. Phys. Rev. B 2015, 91, 241110. [CrossRef]

62. Banerjee, A.; Bridges, C.A.; Yan, J.Q.; Aczel, A.A.; Li, L.; Stone, M.B.; Granroth, G.E.; Lumsden, M.D.; Yiu, Y.; Knolle, J.; et al. Proximate Kitaev quantum spin liquid behaviour in a honeycomb magnet. Nat. Mater. 2016, 15, 733-740. [CrossRef]

63. Cao, H.B.; Banerjee, A.; Yan, J.Q.; Bridges, C.A.; Lumsden, M.D.; Mandrus, D.G.; Tennant, D.A.; Chakoumakos, B.C.; Nagler, S.E. Low-temperature crystal and magnetic structure of $\alpha-\mathrm{RuCl}_{3}$. Phys. Rev. B 2016, 93, 134423. [CrossRef]

64. Biesner, T.; Biswas, S.; Li, W.; Saito, Y.; Pustogow, A.; Altmeyer, M.; Wolter, A.U.B.; Büchner, B.; Roslova, M.; Doert, T.; et al. Detuning the honeycomb of $\alpha-\mathrm{RuCl}_{3}$ : Pressure-dependent optical studies reveal broken symmetry. Phys. Rev. B 2018, 97, 220401. [CrossRef]

65. Sears, J.A.; Songvilay, M.; Plumb, K.W.; Clancy, J.P.; Qiu, Y.; Zhao, Y.; Parshall, D.; Kim, Y.J. Magnetic order in $\alpha-\mathrm{RuCl}_{3}$ : A honeycomb-lattice quantum magnet with strong spin-orbit coupling. Phys. Rev. B 2015, 91, 144420. [CrossRef]

66. Fletcher, J.M.; Gardner, W.E.; Fox, A.C.; Topping, G. X-ray, Infrared, and Magnetic Studies of $\alpha$ - and $\beta$-Ruthenium Trichloride. J. Chem. Soc. A 1967, 0, 1038-1045. [CrossRef]

67. Binotto, L.; Pollini, I.; Spinolo, G. Optical and transport properties of the magnetic semiconductor $\alpha-\mathrm{RuCl}_{3}$. Phys. Stat. Sol. (b) 1971, 44, 245-252. [CrossRef]

68. Guizzetti, G.; Reguzzoni, E.; Pollini, I. Spin-polarized electron tunneling. Phys. Lett. A 1979, 70, 34-36. [CrossRef]

69. Sandilands, L.J.; Tian, Y.; Reijnders, A.A.; Kim, H.S.; Plumb, K.W.; Kim, Y.J.; Kee, H.Y.; Burch, K.S. Spin-orbit excitations and electronic structure of the putative Kitaev magnet $\alpha-\mathrm{RuCl}_{3}$. Phys. Rev. B 2016, 93, 075144. [CrossRef]

70. Sandilands, L.J.; Sohn, C.H.; Park, H.J.; Kim, S.Y.; Kim, K.W.; Sears, J.A.; Kim, Y.J.; Noh, T.W. Optical probe of Heisenberg-Kitaev magnetism in $\alpha-\mathrm{RuCl}_{3}$. Phys. Rev. B 2016, 94, 195156. [CrossRef]

71. Wang, Z.; Guo, J.; Tafti, F.F.; Hegg, A.; Sen, S.; Sidorov, V.A.; Wang, L.; Cai, S.; Yi, W.; Zhou, Y.; et al. Pressure-induced melting of magnetic order and emergence of a new quantum state in $\alpha-\mathrm{RuCl}_{3}$. Phys. Rev. B 2018, 97, 245149. [CrossRef]

72. Cui, Y.; Zheng, J.; Ran, K.; Wen, J.; Liu, Z.X.; Liu, B.; Guo, W.; Yu, W. High-pressure magnetization and NMR studies of $\alpha-\mathrm{RuCl}_{3}$. Phys. Rev. B 2017, 96, 205147. [CrossRef]

73. Bastien, G.; Garbarino, G.; Yadav, R.; Martinez-Casado, F.J.; Beltrán Rodríguez, R.; Stahl, Q.; Kusch, M.; Limandri, S.P.; Ray, R.; Lampen-Kelley, P.; et al. Pressure-induced dimerization and valence bond crystal formation in the Kitaev-Heisenberg magnet $\alpha-\mathrm{RuCl}_{3}$. Phys. Rev. B 2018, 97, 241108. [CrossRef]

74. Kimchi, I.; Analytis, J.G.; Vishwanath, A. Three-dimensional quantum spin liquids in models of harmonic-honeycomb iridates and phase diagram in an infinite-D approximation. Phys. Rev. B 2014, 90, 205126. [CrossRef]

75. Kimchi, I.; Coldea, R.; Vishwanath, A. Unified theory of spiral magnetism in the harmonic-honeycomb iridates $\alpha, \beta$, and $\gamma \mathrm{Li}_{2} \mathrm{IrO}_{3}$. Phys. Rev. B 2015, 91, 245134. [CrossRef]

76. Singh, Y.; Manni, S.; Reuther, J.; Berlijn, T.; Thomale, R.; Ku, W.; Trebst, S.; Gegenwart, P. Relevance of the Heisenberg-Kitaev Model for the Honeycomb Lattice Iridates $\mathrm{A}_{2} \mathrm{IrO}_{3}$. Phys. Rev. Lett. 2012, 108, 127203. [CrossRef]

77. Chaloupka, J.; Jackeli, G.; Khaliullin, G. Kitaev-Heisenberg Model on a Honeycomb Lattice: Possible Exotic Phases in Iridium Oxides $\mathrm{A}_{2} \mathrm{IrO}_{3}$. Phys. Rev. Lett. 2010, 105, 027204. [CrossRef]

78. Gretarsson, H.; Clancy, J.P.; Liu, X.; Hill, J.P.; Bozin, E.; Singh, Y.; Manni, S.; Gegenwart, P.; Kim, J.; Said, A.H.; et al. Crystal-Field Splitting and Correlation Effect on the Electronic Structure of $\mathrm{A}_{2} \mathrm{IrO}_{3}$. Phys. Rev. Lett. 2013, 110, 076402. [CrossRef] 
79. Li, Y.; Foyevtsova, K.; Jeschke, H.O.; Valentí, R. Analysis of the optical conductivity for $\mathrm{A}_{2} \mathrm{IrO}_{3}(\mathrm{~A}=\mathrm{Na}, \mathrm{Li})$ from first principles. Phys. Rev. B 2015, 91, 161101. [CrossRef]

80. Kim, B.H.; Shirakawa, T.; Yunoki, S. From a Quasimolecular Band Insulator to a Relativistic Mott Insulator in $\mathrm{t}_{2 g}^{5}$ Systems with a Honeycomb Lattice Structure. Phys. Rev. Lett. 2016, 117, 187201. [CrossRef]

81. Kim, B.J.; Ohsumi, H.; Komesu, T.; Sakai, S.; Morita, T.; Takagi, H.; Arima, T. Phase-Sensitive Observation of a Spin-Orbital Mott State in $\mathrm{Sr}_{2} \mathrm{IrO}_{4}$. Science 2009, 323, 1329-1332. [CrossRef]

82. Sohn, C.H.; Kim, H.S.; Qi, T.F.; Jeong, D.W.; Park, H.J.; Yoo, H.K.; Kim, H.H.; Kim, J.Y.; Kang, T.D.; Cho, D.Y.; et al. Mixing between $J_{e f f}=\frac{1}{2}$ and $\frac{3}{2}$ orbitals in $\mathrm{Na}_{2} \mathrm{IrO}_{3}$ : A spectroscopic and density functional calculation study. Phys. Rev. B 2013, 88, 085125. [CrossRef]

83. Hermann, V.; Altmeyer, M.; Ebad-Allah, J.; Freund, F.; Jesche, A.; Tsirlin, A.A.; Hanfland, M.; Gegenwart, P.; Mazin, I.I.; Khomskii, D.I.; et al. Competition between spin-orbit coupling, magnetism, and dimerization in the honeycomb iridates: $\alpha-\mathrm{Li}_{2} \mathrm{IrO}_{3}$ under pressure. Phys. Rev. B 2018, 97, 020104. [CrossRef]

84. Williams, S.C.; Johnson, R.D.; Freund, F.; Choi, S.; Jesche, A.; Kimchi, I.; Manni, S.; Bombardi, A.; Manuel, P.; Gegenwart, P.; et al. Incommensurate counterrotating magnetic order stabilized by Kitaev interactions in the layered honeycomb $\alpha-\mathrm{Li}_{2} \mathrm{IrO}_{3}$. Phys. Rev. B 2016, 93, 195158. [CrossRef]

85. Kimber, S.A.J.; Mazin, I.I.; Shen, J.; Jeschke, H.O.; Streltsov, S.V.; Argyriou, D.N.; Valentí, R.; Khomskii, D.I. Valence bond liquid phase in the honeycomb lattice material $\mathrm{Li}_{2} \mathrm{RuO}_{3}$. Phys. Rev. B 2014, 89, 081408. [CrossRef]

86. Clancy, J.P.; Gretarsson, H.; Sears, J.A.; Singh, Y.; Desgreniers, S.; Mehlawat, K.; Layek, S.; Rozenberg, G.K.; Ding, Y.; Upton, M.H.; et al. Pressure-driven collapse of the relativistic electronic ground state in a honeycomb iridate. Npj Quantum Mater. 2018, 3, 35. [CrossRef]

87. Hermann, V.; Ebad-Allah, J.; Freund, F.; Jesche, A.; Tsirlin, A.A.; Gegenwart, P.; Kuntscher, C.A. Optical signature of the pressure-induced dimerization in the honeycomb iridate $\alpha-\mathrm{Li}_{2} \mathrm{IrO}_{3}$. Phys. Rev. B 2019, 99, 235116. [CrossRef]

88. Takayama, T.; Kato, A.; Dinnebier, R.; Nuss, J.; Kono, H.; Veiga, L.S.I.; Fabbris, G.; Haskel, D.; Takagi, H. Hyperhoneycomb Iridate $\beta$ - $\mathrm{Li}_{2} \mathrm{IrO}_{3}$ as a Platform for Kitaev Magnetism. Phys. Rev. Lett. 2015, 114, 077202. [CrossRef]

89. Katukuri, V.M.; Yadav, R.; Hozoi, L.; Nishimoto, S.; van den Brink, J. The vicinity of hyper-honeycomb $\beta$ - $\mathrm{Li}_{2} \mathrm{IrO}_{3}$ to a three-dimensional Kitaev spin liquid state. Sci. Rep. 2016, 6, 29585. [CrossRef]

90. Glamazda, A.; Lemmens, P.; Do, S.H.; Choi, Y.S.; Choi, K.Y. Raman spectroscopic signature of fractionalized excitations in the harmonic-honeycomb iridates $\beta$ - and $\gamma-\mathrm{Li}_{2} \mathrm{IrO}_{3}$. Nat. Commun. 2016, 7, 12286. [CrossRef]

91. Ruiz, A.; Frano, A.; Breznay, N.P.; Kimchi, I.; Helm, T.; Oswald, I.; Chan, J.Y.; Birgeneau, R.J.; Islam, Z.; Analytis, J.G. Correlated states in $\beta-\mathrm{Li}_{2} \mathrm{IrO}_{3}$ driven by applied magnetic fields. Nat. Commun. 2017, 8, 961. [CrossRef]

92. Veiga, L.S.I.; Etter, M.; Glazyrin, K.; Sun, F.; Escanhoela, C.A.; Fabbris, G.; Mardegan, J.R.L.; Malavi, P.S.; Deng, Y.; Stavropoulos, P.P.; et al. Pressure tuning of bond-directional exchange interactions and magnetic frustration in the hyperhoneycomb iridate $\beta-\mathrm{Li}_{2} \mathrm{IrO}_{3}$. Phys. Rev. B 2017, 96, 140402. [CrossRef]

93. Kim, H.S.; Kim, Y.B.; Kee, H.Y. Revealing frustrated local moment model for pressurized hyperhoneycomb iridate: Paving the way toward a quantum spin liquid. Phys. Rev. B 2016, 94, 245127. [CrossRef]

94. Majumder, M.; Manna, R.S.; Simutis, G.; Orain, J.C.; Dey, T.; Freund, F.; Jesche, A.; Khasanov, R.; Biswas, P.K.; Bykova, E.; et al. Breakdown of Magnetic Order in the Pressurized Kitaev Iridate $\beta-\mathrm{Li}_{2} \mathrm{IrO}_{3}$. Phys. Rev. Lett. 2018, 120, 237202. [CrossRef]

95. Modic, K.A.; Smidt, T.E.; Kimchi, I.; Breznay, N.P.; Biffin, A.; Choi, S.; Johnson, R.D.; Coldea, R.; Watkins-Curry, P.; McCandless, G.T.; et al. Realization of a three-dimensional spin-anisotropic harmonic honeycomb iridate. Nat. Commun. 2014, 5, 4203. [CrossRef]

96. Biffin, A.; Johnson, R.D.; Kimchi, I.; Morris, R.; Bombardi, A.; Analytis, J.G.; Vishwanath, A.; Coldea, R. Noncoplanar and Counterrotating Incommensurate Magnetic Order Stabilized by Kitaev Interactions in $\gamma-\mathrm{Li}_{2} \mathrm{IrO}_{3}$. Phys. Rev. Lett. 2014, 113, 197201. [CrossRef]

97. Breznay, N.P.; Ruiz, A.; Frano, A.; Bi, W.; Birgeneau, R.J.; Haskel, D.; Analytis, J.G. Resonant x-ray scattering reveals possible disappearance of magnetic order under hydrostatic pressure in the Kitaev candidate $\gamma-\mathrm{Li}_{2} \mathrm{IrO}_{3}$. Phys. Rev. B 2017, 96, 020402. [CrossRef] 
98. Hermann, V.; Ebad-Allah, J.; Freund, F.; Pietsch, I.M.; Jesche, A.; Tsirlin, A.A.; Deisenhofer, J.; Hanfland, M.; Gegenwart, P.; Kuntscher, C.A. High-pressure versus isoelectronic doping effect on the honeycomb iridate $\mathrm{Na}_{2} \mathrm{IrO}_{3}$. Phys. Rev. B 2017, 96, 195137. [CrossRef]

99. Choi, S.K.; Coldea, R.; Kolmogorov, A.N.; Lancaster, T.; Mazin, I.I.; Blundell, S.J.; Radaelli, P.G.; Singh, Y.; Gegenwart, P.; Choi, K.R.; et al. Spin Waves and Revised Crystal Structure of Honeycomb Iridate $\mathrm{Na}_{2} \mathrm{IrO}_{3}$. Phys. Rev. Lett. 2012, 108, 127204. [CrossRef]

100. Ye, F.; Chi, S.; Cao, H.; Chakoumakos, B.C.; Fernandez-Baca, J.A.; Custelcean, R.; Qi, T.F.; Korneta, O.B.; $\mathrm{Cao}, \mathrm{G}$. Direct evidence of a zigzag spin-chain structure in the honeycomb lattice: A neutron and X-ray diffraction investigation of single-crystal $\mathrm{Na}_{2} \mathrm{IrO}_{3}$. Phys. Rev. B 2012, 85, 180403. [CrossRef]

101. Foyevtsova, K.; Jeschke, H.O.; Mazin, I.I.; Khomskii, D.I.; Valentí, R. Ab initio analysis of the tight-binding parameters and magnetic interactions in $\mathrm{Na}_{2} \mathrm{IrO}_{3}$. Phys. Rev. B 2013, 88, 035107. [CrossRef]

102. Comin, R.; Levy, G.; Ludbrook, B.; Zhu, Z.H.; Veenstra, C.N.; Rosen, J.A.; Singh, Y.; Gegenwart, P.; Stricker, D.; Hancock, J.N.; et al. $\mathrm{Na}_{2} \mathrm{IrO}_{3}$ as a Novel Relativistic Mott Insulator with a 340-meV Gap. Phys. Rev. Lett. 2012, 109, 266406. [CrossRef]

103. Li, Y.; Winter, S.M.; Jeschke, H.O.; Valentí, R. Electronic excitations in $\gamma$-Li $\mathrm{L}_{2} \mathrm{IrO}_{3}$. Phys. Rev. B 2017, $95,045129$. [CrossRef]

104. Kozlenko, D.P.; Kusmartseva, A.F.; Lukin, E.V.; Keen, D.A.; Marshall, W.G.; de Vries, M.A.; Kamenev, K.V. From Quantum Disorder to Magnetic Order in an $s=1 / 2$ Kagome Lattice: A Structural and Magnetic Study of Herbertsmithite at High Pressure. Phys. Rev. Lett. 2012, 108, 187207. [CrossRef]

105. Norman, M.R. Colloquium: Herbertsmithite and the search for the quantum spin liquid. Rev. Mod. Phys. 2016, 88, 041002. [CrossRef]

106. Puphal, P.; Bolte, M.; Sheptyakov, D.; Pustogow, A.; Kliemt, K.; Dressel, M.; Baenitz, M.; Krellner, C. Strong magnetic frustration in $\mathrm{Y}_{3} \mathrm{Cu}_{9}(\mathrm{OH})_{19} \mathrm{Cl}_{8}$ : a distorted kagome antiferromagnet. J. Mater. Chem. C 2017, 5, 2629-2635. [CrossRef]

107. Mendels, P.; Bert, F.; de Vries, M.A.; Olariu, A.; Harrison, A.; Duc, F.; Trombe, J.C.; Lord, J.S.; Amato, A.; Baines, C. Quantum Magnetism in the Paratacamite Family: Towards an Ideal Kagome Lattice. Phys. Rev. Lett. 2007, 98, 077204. [CrossRef]

108. Helton, J.S.; Matan, K.; Shores, M.P.; Nytko, E.A.; Bartlett, B.M.; Yoshida, Y.; Takano, Y.; Suslov, A.; Qiu, Y.; Chung, J.H.; et al. Spin Dynamics of the Spin-1/2 Kagome Lattice Antiferromagnet $\mathrm{ZnCu}_{3}(\mathrm{OH})_{6} \mathrm{Cl}_{2}$. Phys, Rev, Lett, 2007, 98, 107204. [CrossRef]

109. Zorko, A.; Nellutla, S.; van Tol, J.; Brunel, L.C.; Bert, F.; Duc, F.; Trombe, J.C.; de Vries, M.A.; Harrison, A.; Mendels, P. Dzyaloshinsky-Moriya Anisotropy in the Spin-1/2 Kagome Compound $\mathrm{ZnCu}_{3}(\mathrm{OH})_{6} \mathrm{Cl}_{2}$. Phys. Rev. Lett. 2008, 101, 026405. [CrossRef]

110. Jeong, M.; Bert, F.; Mendels, P.; Duc, F.; Trombe, J.C.; de Vries, M.A.; Harrison, A. Field-Induced Freezing of a Quantum Spin Liquid on the Kagome Lattice. Phys. Rev. Lett. 2011, 107, 237201. [CrossRef]

111. Takatsu, K.i.; Shiramura, W.; Tanaka, H. Ground States of Double Spin Chain Systems $\mathrm{TlCuCl}_{3}, \mathrm{NH}_{4} \mathrm{CuCl}_{3}$ and $\mathrm{KCuBr}_{3}$. J. Phys. Soc. Jpn. 1997, 66, 1611-1614. [CrossRef]

112. Oosawa, A.; Ishii, M.; Tanaka, H. Field-induced three-dimensional magnetic orderingin the spin-gap system $\mathrm{TlCuCl}_{3}$. J. Phys. Condens. Matter 1999, 11, 265-271. [CrossRef]

113. Oosawa, A.; Fujisawa, M.; Osakabe, T.; Kakurai, K.; Tanaka, H. Neutron Diffraction Study of the Pressure-Induced Magnetic Ordering in the Spin Gap System TlCuCl 3 . J. Phys. Soc. Jpn. 2003, 72, 1026-1029. [CrossRef]

114. Tanaka, H.; Goto, K.; Fujisawa, M.; Ono, T.; Uwatoko, Y. Magnetic ordering under high pressure in the quantum spin system $\mathrm{TlCuCl}_{3}$. Physica B 2003, 329-333, 697-698. [CrossRef]

115. Goto, K.; Fujisawa, M.; Ono, T.; Tanaka, H.; Uwatoko, Y. Pressure-induced Magnetic Quantum Phase Transition from Gapped Ground State in $\mathrm{TlCuCl}_{3}$. J. Phys. Soc. Jpn. 2004, 73, 3254-3257. [CrossRef]

116. Rüegg, C.; Furrer, A.; Sheptyakov, D.; Strässle, T.; Krämer, K.W.; Güdel, H.U.; Mélési, L. Pressure-Induced Quantum Phase Transition in the Spin-Liquid $\mathrm{TlCuCl}_{3}$. Phys. Rev. Lett. 2004, 93, 257201. [CrossRef]

117. Merchant, P.; Normand, B.; Krämer, K.W.; Boehm, M.; McMorrow, D.F.; Rüegg, C. Quantum and classical criticality in a dimerized quantum antiferromagnet. Nat. Phys. 2014, 10, 373. [CrossRef]

118. Rüegg, C.; Cavadini, N.; Furrer, A.; Güdel, H.U.; Krämer, K.; Mutka, H.; Wildes, A.; Habicht, K.; Vorderwisch, P. Bose-Einstein condensation of the triplet states in the magnetic insulator $\mathrm{TlCuCl}_{3}$. Nature 2003, 423, 62-65. [CrossRef] 
119. Oosawa, A.; Ono, T.; Tanaka, H. Impurity-induced antiferromagnetic ordering in the spin gap system $\mathrm{TlCuCl}_{3}$. Phys. Rev. B 2002, 66, 020405. [CrossRef]

120. Matsumoto, M.; Normand, B.; Rice, T.M.; Sigrist, M. Field- and pressure-induced magnetic quantum phase transitions in $\mathrm{TlCuCl}_{3}$. Phys. Rev. B 2004, 69, 054423. [CrossRef]

121. Rüegg, C.; Normand, B.; Matsumoto, M.; Furrer, A.; McMorrow, D.F.; Krämer, K.W.; Güdel, H.U.; Gvasaliya, S.N.; Mutka, H.; Boehm, M. Quantum Magnets under Pressure: Controlling Elementary Excitations in $\mathrm{TlCuCl}_{3}$. Phys. Rev. Lett. 2008, 100, 205701. [CrossRef] [PubMed]

(C) 2019 by the authors. Licensee MDPI, Basel, Switzerland. This article is an open access article distributed under the terms and conditions of the Creative Commons Attribution (CC BY) license (http:// creativecommons.org/licenses/by/4.0/). 